\title{
Frictional drag between quantum wells mediated by phonon exchange
}

\author{
Bønsager, M.C.; Flensberg, Karsten; Hu, Ben Yu-Kuang; MacDonald, A.H.
}

\section{Published in:}

Physical Review B

Link to article, DOI:

10.1103/PhysRevB.57.7085

Publication date:

1998

Document Version

Publisher's PDF, also known as Version of record

Link back to DTU Orbit

Citation (APA):

Bønsager, M. C., Flensberg, K., Hu, B. Y-K., \& MacDonald, A. H. (1998). Frictional drag between quantum wells mediated by phonon exchange. Physical Review B, 57(12), 7085-7102.

https://doi.org/10.1103/PhysRevB.57.7085

\section{General rights}

Copyright and moral rights for the publications made accessible in the public portal are retained by the authors and/or other copyright owners and it is a condition of accessing publications that users recognise and abide by the legal requirements associated with these rights.

- Users may download and print one copy of any publication from the public portal for the purpose of private study or research.

- You may not further distribute the material or use it for any profit-making activity or commercial gain

- You may freely distribute the URL identifying the publication in the public portal

If you believe that this document breaches copyright please contact us providing details, and we will remove access to the work immediately and investigate your claim. 


\title{
Frictional drag between quantum wells mediated by phonon exchange
}

\author{
Martin Chr. Bønsager \\ Department of Physics, Indiana University, Bloomington, Indiana 47405 \\ Karsten Flensberg \\ Danish Institute of Fundamental Metrology, Building 307, Anker Engelunds Vej 1, DK-2800 Lyngby, Denmark \\ Ben Yu-Kuang $\mathrm{Hu}$ \\ Mikroelektronik Centret, Building 345 east, Technical University of Denmark, DK-2800 Lyngby, Denmark \\ A. H. MacDonald \\ Department of Physics, Indiana University, Bloomington, Indiana 47405
}

(Received 10 July 1997)

\begin{abstract}
We use the Kubo formalism to evaluate the contribution of acoustic-phonon exchange to the frictional drag between nearby two-dimensional electron systems. In the case of free phonons, we find a divergent drag rate $\left(\tau_{D}^{-1}\right)$. However, $\tau_{D}^{-1}$ becomes finite when phonon scattering from either lattice imperfections or electronic excitations is accounted for. In the case of GaAs quantum wells, we find that for a phonon mean free path $\ell_{\mathrm{ph}}$ smaller than a critical value, imperfection scattering dominates and the drag rate varies as $\ln \left(\ell_{\mathrm{ph}} / d\right)$ over many orders of magnitude of the layer separation $d$. When $\ell_{\mathrm{ph}}$ exceeds the critical value, the drag rate is dominated by coupling through an electron-phonon collective mode localized in the vicinity of the electron layers. We argue that the coupled electron-phonon mode may be observable for realistic parameters. Our theory is in good agreement with experimental results for the temperature, density, and $d$ dependence of the drag rate.
\end{abstract} [S0163-1829(98)03612-1]

\section{INTRODUCTION}

Interactions between particles are a cornerstone of much of today's research in physics. In nuclear and high-energy physics, the effects of these interactions can be probed directly through scattering experiments. In condensed-matter physics, interparticle interaction effects are enriched by the close proximity of other particles giving rise to a plethora of fascinating phenomena. However, direct measurement of these interactions in a condensed-matter system is often a more difficult exercise, because of the indirect way in which scattering amplitudes are related to observables.

Some time ago, Pogrebinskii and later Price ${ }^{1}$ proposed the following direct probe of interparticle interactions through a transport measurement. Place two two-dimensional (2D) electron films close enough together and draw a current in one film. Through interlayer interactions, net momentum is transferred to electrons in the adjacent film, inducing a current there which can be measured. Due to technological difficulties in contacting the individual layers, decades passed before the first frictional drag experiment between 2D and three-dimensional (3D) layers was performed. ${ }^{2}$ The first experiments on this phenomenon between two 2D systems, as originally envisaged in Ref. 1, were performed by Gramila et al. for two electron layers, ${ }^{3,4}$ and by Sivan, Solomon, and Shtrikman for an electron-hole system. ${ }^{5}$ In these experiments a current is drawn in the first layer, while the second layer is an open circuit. Instead of a current in the second layer, there will be an induced electric field that opposes the "dragging force" from the first layer. The transresistivity $\stackrel{\leftrightarrow}{\rho}_{21}$ is defined as the ratio of the induced electric field in the second layer to the driving current density in the first,

$$
\stackrel{\leftrightarrow}{\rho}_{21} \cdot \mathbf{J}_{1}=\mathbf{E}_{2} .
$$

The stronger the interlayer interaction, the larger the magnitude of the transresistivity. (In this paper, we shall treat isotropic systems at zero magnetic field, hence $\stackrel{\leftrightarrow}{\rho}_{21}$ is diagonal). The transresistivity is often interpreted in terms of a drag rate which, in analogy with a Drude model, is defined by $\tau_{D}^{-1}$ $=\rho_{21} n_{1} e^{2} / \mathrm{m}^{*}$, where $n_{1}$ is the electron density of the driving layer and $m^{*}$ is the electron effective mass.

These experiments spurred a large body of theoretical work both on electron-hole systems ${ }^{6}$ and on electron-electron systems. ${ }^{7-17}$ Most of this work focused on interlayer Coulomb interaction, the most obvious coupling mechanism and the one considered in the original theoretical papers. ${ }^{1}$ However, it was clear from the start that the experimental results were inconsistent with a purely Coulomb interlayer interaction, which predicted a low-temperature ${ }^{3,11,12}$ $\left(k_{B} T \ll \varepsilon_{F, 1}, \varepsilon_{F, 2}\right.$, where $\varepsilon_{F, i}$ is the Fermi energy for layer $\left.i\right)$ transresistivity of the form

$\rho_{21}=\left(-\frac{h}{e^{2}}\right) \frac{\zeta(3) \pi}{32} \frac{\left(k_{B} T\right)^{2}}{\varepsilon_{F, 1} \varepsilon_{F, 2}} \frac{1}{\left(k_{F, 1} d\right)\left(k_{F, 2} d\right)} \frac{1}{\left(q_{\mathrm{TF}} d\right)\left(q_{\mathrm{TF}} d\right)}$,

where $\zeta$ is the Riemann zeta function, $d$ is the interlayer separation, $k_{F, i}$ is the Fermi wave vector for layer $i$, and $q_{\mathrm{TF}}$ is the Thomas-Fermi screening wave vector of the $2 \mathrm{D}$ electron gas. This expression is based on the random-phase approximation (RPA) for the screened interlayer Coulomb interaction and applies for $q_{\mathrm{TF}} d \gg 1$ and $k_{F, i} d \gg 1$. 
From inspection of Eq. (2), one notes three important characteristics of the Coulomb drag for low temperatures: (1) the scaled transresistivity $\rho_{21}(T) / T^{2}$ is a constant; (2) $\rho_{21}$ is a monotonically decreasing function of the density of either layer (so long as $k_{F, i} d \gg 1$ ); and (3) $\rho_{21} \propto d^{-4}$. The experimental results at around $2-3 \mathrm{~K}$, on the other hand, showed $^{3,4,18}$ (1) a well-defined peak in the $\rho_{21}(T) / T^{2},(2)$ a local maximum in $\rho_{21}$ near equal layer densities and (3) an approximately $d$-independent residual $\rho_{21}$, after subtraction of the Coulomb contribution which can be identified experimentally by its simple $d$ and $T$ dependencies. Furthermore, the experimentally measured magnitude of $\rho_{21}$ was generally larger by about a factor of 2 than the value predicted by the Coulomb interaction alone. Another momentum-transfer mechanism was clearly involved.

From the outset it was understood that exchange of acoustic phonons was the most likely candidate for this second momentum-transfer mechanism. Exchange of phonons often dominates electron-electron scattering contributions to the resistance of bulk metals. ${ }^{19}$ The peak in the temperature dependence of $\rho_{21}(T) / T^{2}$ is reminiscent of features in the temperature dependence of the acoustic-phonon-limited mobility, and occurs around the Bloch-Grüneisen temperature $T_{\mathrm{BG}}=2 k_{B}^{-1} \hbar c_{l} k_{F}$ associated with the acoustic-phonon modes. ( $c_{l}$ is the longitudinal acoustic-phonon velocity.) The phase space available for scattering is largest for $2 k_{F}$ transfers, partially explaining the enhanced drag when $k_{F, 1}$ $=k_{F, 2}$. Finally, the long-ranged exchange of phonons between electrically isolated systems is not an unknown phenomenon. Exchange of phonons between two 3D systems separated by $\sim 100 \mu \mathrm{m}$ was observed previously, ${ }^{20}$ and related effects are expected to be observable in a superlattice if the driving electron layer is hot. ${ }^{21}$ The theoretical challenge is to explain the magnitude of the observed drag and its dependence on layer separation and density.

Since interactions of acoustic phonons with electrons are relatively weak in GaAs, the fact that phonon-mediated and Coulomb contribution to the drag are often comparable seems mysterious. However, we show below that the obvious calculation, in which a free-phonon propagator substitutes for the Coulomb interaction, leads to a divergent drag resistivity. The large but finite drag rates which are observed experimentally can be explained in terms of scattering and interaction effects which alter the phonon propagator.

Despite the apparent importance of phonon exchange in drag measurements, there has been less theoretical work on this mechanism than on the Coulomb coupling mechanism. An incoherent phonon exchange model studied by Gramila et al. produced a drag rate which was too weak to account for the observed transresistivity. ${ }^{4}$ Tso, Vasilopoulos, and Peeters ${ }^{9}$ addressed the question of whether exchange of virtual phonons could make a contribution strong enough to explain the magnitude of the transresistivity, but did not use an electron-phonon coupling model which is realistic for $\mathrm{GaAs} / \mathrm{Al}_{x} \mathrm{Ga}_{1-x} \mathrm{As}$ systems. Zhang and Takahashi ${ }^{10}$ included all relevant phonon contributions; however, due to an incorrect effective two-dimensional electron-phonon interaction, they predicted a short-range phonon-mediated drag. In spite of the use of radically different models for the phononmediated process, both these calculations yielded a temperature dependence for the drag in reasonable agreement with experiment. The similarity is not surprising because of the common appearance of the Bloch-Grüneisen temperature scale associated with the acoustic-phonon mode. Comparable features in the drag, associated with plasmon modes of the electronic system, were predicted ${ }^{14,16}$ and observed ${ }^{22,23}$ at higher temperatures.

In this paper we report on a detailed examination of the phonon exchange mechanism for drag, using a model which we believe to be quantitatively reliable for GaAs/AlAs quantum-well systems. We address the distance, temperature, and density dependence of the transresistivity. We show that two different regimes of layer separation dependence can occur, depending on the phonon mean free path and the electron-phonon coupling constant. We demonstrate the existence of a coupled electron-phonon mode, which for long phonon mean free paths leads to a large enhancement of the drag.

The outline of the paper is as follows. In Sec. II we use a Kubo linear-response formalism to obtain a formula for the transresistivity which is sufficiently general to permit the incorporation of a finite phonon mean free path and renormalization of the phonon propagator due to coupling to the electronic layers. In Sec. III we discuss the phonon-mediated $e-e$ interaction, and explain how the relatively weak electronphonon interaction can lead to a surprisingly large contribution to the drag. The two different regimes of $\ell_{\mathrm{ph}}$ are discussed in Secs. IV and V, where approximate expressions for $\rho_{21}$ are derived, and special attention is paid to the layer separation dependence of the drag. Detailed numerical results are presented in Sec. VI before we conclude in Sec. VII with a summary of results.

\section{FORMALISM}

Identical theoretical expressions for the drag rate due to Coulomb interactions were obtained in several different ways. The most physically transparent derivation was based on semiclassical transport theory. ${ }^{11}$ More elaborate fully quantum mechanical-derivations based on memory function $^{12}$ or Kubo formula approaches ${ }^{13,15}$ yield identical results for the large $k_{F} \ell$ limit at zero magnetic field but are more flexible and, in particular, can be applied in the presence of an external magnetic field. (Here $\ell$ is the electronic mean free path.) We show below that at lowest nonvanishing order in the electron-phonon interaction, phonon exchange yields an infinite result for the drag rate. The Kubo formula approach, which we use in this paper, is most convenient when interlayer interactions need to be treated beyond leading order, because of the powerful diagrammatic perturbation theory expansion available to evaluate the influence of interaction terms on the appropriate current-current correlation function. The required calculation is an adaptation of those described in Refs. 13 and 15, and is outlined below.

\section{A. Electron-phonon interaction Hamiltonian}

We consider frictional drag between two GaAs quantum wells. We define the plane of the quantum wells as the $x-y$ plane. The distance between the centers of the two quantum wells is $d$, and the width of the two wells is $L$. We assume that the electron number densities in each well, $n_{1}$ and $n_{2}$, are such that only one subband of the quantum well is occu- 
pied. The formalism can easily be generalized to accommodate more occupied subbands.

We include interlayer and intralayer Coulombic electronelectron interactions and the coupling of electrons in either layer to the $3 \mathrm{D}$ phonons of the semiconductor host. ${ }^{24} \mathrm{We}$ denote $3 \mathrm{D}$ wave vectors by upper case letters, and their projection onto the $x-y$ plane by the corresponding lower case letter so that $\mathbf{Q}=\left(\mathbf{q}, Q_{z}\right)$. The electron creation (annihilation) operator in layer $i$ is $\hat{c}_{i}^{\dagger}(\mathbf{k})\left[\hat{c}_{i}(\mathbf{k})\right]$ with implicit spin indices, the phonon creation (annihilation) operator for polarization $\lambda$ is $\hat{a}_{\lambda, \mathbf{Q}}^{\dagger}\left(\hat{a}_{\lambda, \mathbf{Q}}\right)$, and the subband wave function of electrons in well $i$ is $\varphi_{i}(z)$. With these definitions, the electron-phonon interaction contribution to the Hamiltonian is given by

$$
\begin{aligned}
\hat{H}_{e-\mathrm{ph}}= & V^{-1 / 2} \sum_{\lambda} \sum_{i=1,2} \sum_{\mathbf{q}, Q_{z}} M_{\lambda}\left(\mathbf{q}, Q_{z}\right) \hat{\mathcal{A}}_{\lambda, \mathbf{q}, Q_{z}} \hat{\rho}_{i}(-\mathbf{q}) \\
& \times F_{i}\left(Q_{z}\right),
\end{aligned}
$$

where $V$ is the normalization volume,

$$
\begin{gathered}
F_{i}\left(Q_{z}\right)=\int_{-\infty}^{\infty} d z\left|\varphi_{i}(z)\right|^{2} e^{-i Q_{z} z}, \\
\hat{\mathcal{A}}_{\lambda, \mathbf{Q}}=\hat{a}_{\lambda, \mathbf{Q}}+\hat{a}_{\lambda,-\mathbf{Q}}^{\dagger}, \\
\rho_{i}(\mathbf{q})=\sum_{\mathbf{k}} \hat{c}_{i}^{\dagger}(\mathbf{k}) \hat{c}_{i}(\mathbf{k}+\mathbf{q}),
\end{gathered}
$$

and $M_{\lambda}(\mathbf{Q})$ is the bulk electron-phonon coupling constant. At temperatures much lower than the Debye temperature, one can neglect the Umklapp process in the electron-phonon interaction Hamiltonian.

\section{B. Kubo formula transconductivity}

The Kubo formula for linear response offers an expression for the transconductivity tensor which is defined by

$$
\mathbf{J}_{2}=\stackrel{\leftrightarrow}{\sigma}_{21} \mathbf{E}_{1}
$$

In the absence of magnetic fields, $\mathbf{J}_{2}$ and $\mathbf{E}_{1}$ will be antiparallel, and $\stackrel{\leftrightarrow}{\sigma}_{21}$ is a diagonal $2 \times 2$ tensor.

The derivation sketched below for $\sigma_{21}$ is very similar to the one given previously in Ref. 15 , in which the reader can find further details. The transconductivity is given by

$$
\sigma_{21}^{\alpha \gamma}(\mathbf{k}, \Omega)=\frac{i e^{2}}{\hbar \Omega} \Pi_{21}^{\alpha \gamma, \mathrm{ret}}(\mathbf{k}, \Omega),
$$

where $\Pi_{21}^{\alpha \gamma, \text { ret }}(\mathbf{k}, \Omega)$ is the Fourier transform of the retarded current-current correlation function, and $\alpha$ and $\gamma$ are Cartesian indices. The retarded correlation function is evaluated by the standard analytic continuation of the (bosonic Matsubara frequency) Fourier components of its imaginary time counterpart. ${ }^{15}$ The imaginary time correlation function is calculated in perturbation theory

$$
\Pi_{21}^{\alpha \gamma}\left(\mathbf{x}-\mathbf{x}^{\prime}, \tau-\tau^{\prime}\right)=-\frac{\left\langle T_{\tau}\left\{S(\beta) j_{2}^{\alpha}(\mathbf{x}, \tau) j_{1}^{\gamma}\left(\mathbf{x}^{\prime}, \tau^{\prime}\right)\right\}\right\rangle_{0}}{\langle S(\beta)\rangle_{0}},
$$

$$
S(\beta)=T_{\tau}\left\{\exp \left[-\frac{1}{\hbar} \int_{0}^{\hbar \beta} d \tau H_{\text {int }}(\tau)\right]\right\}
$$

where $\langle\cdots\rangle_{0}$ denotes a noninteracting system thermal average, and $T_{\tau}\{\cdots\}$ is the usual $\tau$-ordering operator. The $S$ matrix is expanded in powers of the interaction Hamiltonian, and the resulting noninteracting system correlation functions are evaluated with the aid of Wick's theorem. As usual, the denominator in this expression cancels the "disconnected" terms in the diagrammatic expansion.

If we include only electron-phonon interactions for the moment, the lowest nonvanishing term appears at fourth order, and makes the following contribution to the currentcurrent correlation function:

$$
\begin{aligned}
\Pi_{21}^{\alpha \beta}\left(\mathbf{k}=0, i \Omega_{n}\right)^{(4)}= & -\frac{V^{-2} A^{-1} \hbar^{-4}}{4} \int_{0}^{\beta} d \tau \int_{0}^{\beta} d \tau_{1} \int_{0}^{\beta} d \tau_{2} \int_{0}^{\beta} d \tau_{3} \int_{0}^{\beta} d \tau_{4} \exp \left(i \Omega_{n} \tau\right) \sum_{\mathbf{Q}_{1}, \lambda_{1}} \sum_{\mathbf{Q}_{2}, \lambda_{2}} \sum_{\mathbf{Q}_{3}, \lambda_{3}} \sum_{\mathbf{Q}_{4}, \lambda_{4}} M_{\lambda_{1}}\left(\mathbf{Q}_{1}\right) \\
& \times M_{\lambda_{2}}\left(\mathbf{Q}_{2}\right) M_{\lambda_{3}}\left(\mathbf{Q}_{3}\right) M_{\lambda_{4}}\left(\mathbf{Q}_{4}\right)\left\langle T_{\tau} \hat{j}_{1}^{\alpha}(\mathbf{q}=0, \tau) \hat{\rho}_{1}\left(-\mathbf{q}_{1}, \tau_{1}\right) \hat{\rho}_{1}\left(-\mathbf{q}_{2}, \tau_{2}\right)\right\rangle_{0} \\
& \times\left\langle T_{\tau} \hat{j}_{1}^{\beta}(\mathbf{q}=0,0) \hat{\rho}_{2}\left(-\mathbf{q}_{3}, \tau_{3}\right) \hat{\rho}_{2}\left(-\mathbf{q}_{4}, \tau_{4}\right)\right\rangle_{0} F_{1}\left(Q_{z, 1}\right) F_{1}\left(Q_{z, 2}\right) F_{2}\left(Q_{z, 3}\right) F_{2}\left(Q_{z, 4}\right) \\
& \times\left\langle T_{\tau} \hat{\mathcal{A}}_{\mathbf{Q}_{1}, \lambda_{1}}\left(\tau_{1}\right) \hat{\mathcal{A}}_{\mathbf{Q}_{2}, \lambda_{2}}\left(\tau_{2}\right) \hat{\mathcal{A}}_{\mathbf{Q}_{3}, \lambda_{3}}\left(\tau_{3}\right) \hat{\mathcal{A}}_{\mathbf{Q}_{4}, \lambda_{4}}\left(\tau_{4}\right)\right\rangle_{0}
\end{aligned}
$$




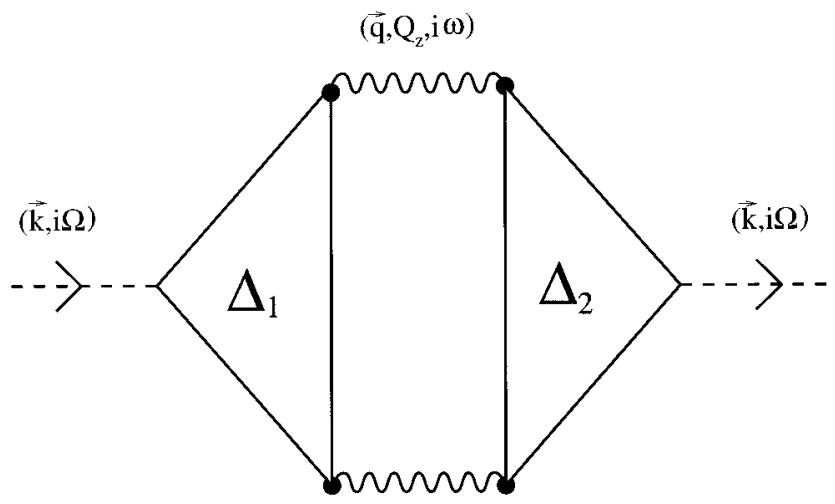

$\left(\vec{k}+\vec{q}, Q_{z}^{\prime}, i \Omega+i \omega\right)$

FIG. 1. The Feynmann diagram corresponding to the correlation function (11). The triangles (the function $\Delta$ ) in each layer are connected by phonon propagators (wiggly lines). The dots represent the $e$-ph coupling, and the dashed lines are external current operators. The frequencies and two-dimensional wave vectors $\mathbf{q}$ are conserved in each vertex, whereas the perpendicular components $Q_{z}$ and $Q_{z}^{\prime}$ are independently integrated over.

where $A$ is the $2 \mathrm{D}$ system area.

The Wick's theorem factorization of the phonon operator product expectation value leads to the product of two bare phonon Green's functions, defined by

$$
D_{\lambda}^{(0)}\left(\mathbf{Q}, \tau-\tau^{\prime}\right)=-\left\langle T_{\tau}\left\{\hat{\mathcal{A}}_{\lambda, \mathbf{Q}}(\tau) \hat{\mathcal{A}}_{\lambda,-\mathbf{Q}}\left(\tau^{\prime}\right)\right\}\right\rangle_{0}
$$

It follows that, to leading order in electron-phonon interactions,

$$
\begin{aligned}
\Pi_{21}^{\alpha \gamma}(\mathbf{k}=0, i \Omega)= & \frac{-1}{2 A \hbar^{2}} \sum_{\mathbf{q}} \frac{1}{\hbar \beta} \sum_{i \omega} \Delta_{2}^{\alpha}(-\mathbf{q},-\mathbf{q},-i \omega \\
& -i \Omega,-i \omega) \Delta_{1}^{\gamma}(\mathbf{q}, \mathbf{q}, i \omega+i \Omega, i \omega) \\
& \times \int \frac{d Q_{z}}{2 \pi \hbar} \sum_{\lambda} F_{1}\left(Q_{z}\right) F_{2}\left(-Q_{z}\right) \\
& \times\left|M_{\lambda}\left(\mathbf{q}, Q_{z}\right)\right|^{2} D_{\lambda}^{(0)}\left(\mathbf{q}, Q_{z}, i \omega\right) \int \frac{d Q_{z}^{\prime}}{2 \pi \hbar} \\
& \times \sum_{\lambda^{\prime}} F_{1}\left(Q_{z}^{\prime}\right) F_{2}\left(-Q_{z}^{\prime}\right)\left|M_{\lambda^{\prime}}\left(\mathbf{q}, Q_{z}^{\prime}\right)\right|^{2} \\
& \times D_{\lambda^{\prime}}^{(0)}\left(\mathbf{q}, Q_{z}^{\prime}, i \Omega+i \omega\right),
\end{aligned}
$$

where

$$
\begin{aligned}
\Delta\left(\mathbf{q}, \mathbf{q} ; i \omega_{n}, i \omega_{n}^{\prime}\right) \equiv & -A^{-1} \int_{0}^{\beta} d \tau_{1} \int_{0}^{\beta} d \tau_{2}\left\langle T_{\tau} \hat{\mathbf{j}}(\mathbf{q}=0,0)\right. \\
& \left.\times \hat{\rho}\left(\mathbf{q}, \tau_{1}\right) \hat{\rho}\left(-\mathbf{q},-\tau_{2}\right)\right\rangle \exp \left(i \omega_{n} \tau_{1}\right) \\
& \times \exp \left(i \omega_{n}^{\prime} \tau_{2}\right)
\end{aligned}
$$

The Feynman diagram corresponding to this contribution to the correlation function (11) is shown in Fig. 1.
It will turn out to be important to account for disorder and anharmonicity in the lattice system. We will do so using a phenomenological approach by introducing a phonon mean free path $\ell_{\mathrm{ph}}$. However, since the intrinsic bulk phonon mean free path may exceed the dimensions of the sample, we should in principle take the surface scattering explicitly into account. For simplicity we will nevertheless use a single phenomenological mean free path and later, when we discuss the long mean free path limit in more detail, take boundary effects into account. The phonon Green's function is then ${ }^{25}$

$$
D_{\lambda}\left(\mathbf{Q}, i \omega_{n}\right)=-\frac{2 \omega_{\lambda, \mathbf{Q}}}{\left[\omega_{n}+\left(c_{\lambda} / 2 \ell_{\mathrm{ph}}\right) \operatorname{sgn}\left(\omega_{n}\right)\right]^{2}+\omega_{\lambda, \mathbf{Q}}^{2}},
$$

where $\omega_{\lambda, \mathbf{Q}}=c_{\lambda} \sqrt{q^{2}+Q_{z}^{2}}$, and $\ell_{\mathrm{ph}}$ is the phonon mean free path.

Note that the wave-vector arguments in $\boldsymbol{\Delta}_{i}$ are $2 \mathrm{D}$, since $\boldsymbol{\Delta}_{i}$ is a property of the $2 \mathrm{D}$ electron systems. One can therefore sum over $\lambda$ and integrate over $Q_{z}$ to obtain a phononmediated effective interaction

$$
\begin{aligned}
\mathcal{D}_{i j}\left(\mathbf{q}, i \omega_{n}\right)= & \int \frac{d Q_{z}}{2 \pi \hbar} \sum_{\lambda}\left|M_{\lambda}(\mathbf{Q})\right|^{2} F_{i}\left(Q_{z}\right) F_{j}\left(-Q_{z}\right) \\
& \times D_{\lambda}\left(\mathbf{Q}, i \omega_{n}\right) .
\end{aligned}
$$

This effective interaction is the 2D Fourier transform of the product of the phonon propagator between the layers and the electron-phonon interaction in each layer. With this definition, Eq. (1) becomes

$$
\begin{aligned}
\Pi_{21}^{\alpha \gamma}(\mathbf{k}=0, i \Omega)= & \frac{-1}{2 A \hbar^{2}} \sum_{\mathbf{q}} \frac{1}{\hbar \beta} \\
& \times \sum_{i \omega} \Delta_{2}^{\alpha}(-\mathbf{q},-\mathbf{q} ;-i \omega-i \Omega,-i \omega) \\
& \times \Delta_{1}^{\gamma}(\mathbf{q}, \mathbf{q} ; i \omega+i \Omega, i \omega) \mathcal{D}_{21}\left(\mathbf{q}, i \omega_{n}\right) \\
& \times \mathcal{D}_{21}\left(\mathbf{q}, i \Omega_{n}+i \omega_{n}\right)
\end{aligned}
$$

This expression for $\Pi_{21}$ is the same as in Ref. 15, except that the interlayer Coulomb interaction is replaced by the phonon-mediated effective interaction. From this point on, the formal steps are identical to the Coulomb case. Performing the summation over $i \omega$, continuing to real frequencies and taking the $\Omega \rightarrow 0$ limit, we obtain ${ }^{15}$

$$
\begin{aligned}
\sigma_{21}^{\alpha \gamma}= & \frac{e^{2}}{2 \hbar^{3} A} \sum_{\mathbf{q}} \int_{-\infty}^{\infty} \frac{d \omega}{2 \pi}\left|\mathcal{D}_{21}(\mathbf{q}, \omega+i \delta)\right|^{2}\left[-\frac{\partial n_{B}(\omega)}{\partial \omega}\right] \\
& \times \Delta_{2}^{\gamma}(-\mathbf{q},-\mathbf{q},-\omega-i \delta,-\omega+i \delta) \\
& \times \Delta_{1}^{\alpha}(\mathbf{q}, \mathbf{q}, \omega+i \delta, \omega-i \delta)
\end{aligned}
$$

\section{From transconductivity to transresistivity}

For further progress it is necessary to make some assumptions about the electronic systems. We will assume that the 2D electron layers are good metals with large $k_{F} \ell$, where $\ell$ is the electronic mean free path. It can then be shown ${ }^{13,15,16}$ 
that whenever the transport scattering time $\tau_{\text {tr }}$ is independent of energy, the function $\Delta$ is related to the electron polarization function $\chi(\mathbf{q}, \omega)$ by

$$
\Delta_{i}^{\alpha}=\frac{2 \tau_{\mathrm{tr}, i}}{m^{*}} q^{\alpha} \operatorname{Im} \chi_{i}(\mathbf{q}, \omega) .
$$

Here $m^{*}$ is the electron effective mass. Relation (17) is a property of the $2 \mathrm{D}$ electron layers only, and is not dependent on the phonon degrees of freedom. Assuming further that $\left|\sigma_{12}\right| \ll \sigma_{i i}$, the transresistivity can be approximated as follows:

$$
\rho_{21}=\frac{-\sigma_{21}}{\sigma_{11} \sigma_{22}-\sigma_{12} \sigma_{21}} \approx \frac{-\sigma_{21}}{\sigma_{11} \sigma_{22}} .
$$

Using the Drude expression $\left(\sigma_{i i}=e^{2} n_{i} \tau_{\operatorname{tr}_{i}} / m^{*}\right)$ for the intralayer conductivities, which is valid under the above assumptions, the transresistivity due to the electron-phonon interaction is given by the following explicit expression;

$$
\begin{aligned}
\rho_{21}= & \frac{-\hbar^{2}}{4 e^{2} n_{1} n_{2} k_{B} T} \frac{1}{A} \sum_{\mathbf{q}} q^{2} \\
& \times \int_{-\infty}^{\infty} \frac{d \omega}{2 \pi}\left|\mathcal{D}_{21}(\mathbf{q}, \omega)\right|^{2} \frac{\operatorname{Im} \chi_{1}(\mathbf{q}, \omega) \operatorname{Im} \chi_{2}(\mathbf{q}, \omega)}{\sinh ^{2}\left(\hbar \omega / 2 k_{B} T\right)} .
\end{aligned}
$$

The same expression for the phonon-exchange contribution to the drag can also be derived using semiclassical Boltzmann transport theory and a collision term with transition matrix elements calculated by summing over virtual and real intermediate states with absorbed and emitted phonons. Ambiguities can arise in that approach, however, from the portion of phase space where the relevant energy denominators approach zero. As we explain below this part of the phase space is important in determining the drag resistivity. Our Kubo function derivation allows finite phonon mean free paths, which remove any spurious singularities, to be incorporated into the calculation in a consistent and unambiguous manner.

Note that the explicit dependence of the transconductivity on the transport lifetime is absent in the transresistivity. (An implicit dependence remains through the dependence of the polarization function on disorder.) This aspect of the final expression, emphasized in recent work by Swierkowski, Szymański and Gortel, ${ }^{17}$ is not accidental, and emerges naturally in a force-balance approximation where the drag force simply cancels the rate of momentum transfer per particle from the current carrying layer to the open layer.

\section{Coulomb interaction and screening}

In reality, electron-electron and electron-phonon interactions are simultaneously present, and both should be included in a drag calculation. To leading order, Coulomb interactions can be incorporated by simply adding ${ }^{26}$ the interlayer Coulomb interaction to the phonon-mediated effective interaction in Eq. (19). One class of higher-order terms in which intralayer Coulomb interactions appear is captured by replacing the electronic polarization functions which appear in Eq. (19) by their interacting system coun- terparts. However, the most essential higher-order terms are those which account for the screening of both phononmediated and Coulombic interactions. In the RPA, the total interlayer screened interaction is given by

$$
W_{21}^{\text {total }}(q, \omega)=\frac{\mathcal{D}_{21}(q, \omega)+U_{21}(q)}{\epsilon(q, \omega)},
$$

where $U_{i j}(q)$ is the unscreened Coulomb interaction, and

$$
\begin{aligned}
\epsilon(q, \omega)= & {\left[1-\left(\mathcal{D}_{11}+U_{11}\right) \chi_{1}\right]\left[1-\left(\mathcal{D}_{22}+U_{22}\right) \chi_{2}\right] } \\
& -\left(\mathcal{D}_{21}+U_{21}\right)^{2} \chi_{1} \chi_{2} .
\end{aligned}
$$

(See, for example, Ref. 10. The form of the phonon-mediated interlayer interaction in this reference is incorrect, however.) $\epsilon(q, \omega)$ is the effective dielectric function for interlayer interactions in the RPA. Notice that when the contribution of interlayer interactions to the screening can be neglected, $\epsilon$ is simply the product of the dielectric functions for the two layers, corresponding to independently screened electronphonon interactions in each layer.

Coulomb and phonon-mediated interactions can be simultaneously included in the transresistivity simply by replacing $\mathcal{D}$ by $W^{\text {total }}$ in Eq. (19). Note that the transresistivity is not strictly the sum of purely "Coulomb" and "phonon" contributions, since there are interference terms proportional to $U \times \mathcal{D}$ in the $\left|W_{21}^{\text {total }}\right|^{2}$. However, the Coulomb contribution is large only for $q \lesssim 0.5 k_{F}\left[U(q)=2 \pi e^{2} \exp (-q d) / q\right.$ when the finite thickness of the electron layers is neglected], whereas contributions from the $\mathcal{D}$ term come predominantly from $q$ $\approx 2 k_{F}$. Hence the interference terms are usually negligible, and in practice we will treat the Coulomb and phonon contributions as if they were incoherent. In what follows, we will concentrate on the "phonon contribution," which will be calculated from Eq. (19), with only the $\mathcal{D}$ term in the numerator,

$$
W_{21}(q, \omega)=\frac{\mathcal{D}_{21}}{\epsilon} .
$$

Note that it is important to retain the coupling between the Coulomb and phonon terms in $\epsilon$, since this can influence the $q \approx 2 k_{F}$ contribution to the transresistivity.

\section{PHONON-MEDIATED INTERACTION IN GaAs/Al ${ }_{X} \mathbf{G a}_{1-X}$ As SYSTEMS}

As mentioned earlier, the acoustic-phonon-electron interaction is weak in GaAs and $\mathrm{Al}_{x} \mathrm{Ga}_{1-x} \mathrm{As}$. In this section we discuss quantitatively what "weak' means, and explain how phonons can make an important contribution despite this weakness. In Sec. IV we discuss how and when screening affects the phonon-mediated interaction.

\section{A. Electron-phonon coupling in $\mathbf{G a A s} / \mathrm{Al}_{x} \mathbf{G a}_{1-x} \mathrm{As}$}

In $\mathrm{GaAs} / \mathrm{Al}_{x} \mathrm{Ga}_{1-x} \mathrm{As}$ systems, electrons couple to acoustic phonons via deformation potential and piezoelectric couplings. Since we are concerned with low-energy excitations, only acoustic phonons in the long-wavelength limit have to 
be considered. In this limit, the squares of the $e$-ph coupling strengths for longitudinal and transverse phonons are, ${ }^{27}$ respectively,

$$
\begin{gathered}
\left|M_{l}(\mathbf{Q})\right|^{2}=\frac{\hbar Q}{2 \varrho c_{l}}\left[D^{2}+\frac{\left(e h_{14}\right)^{2}}{Q^{2}} A_{l}(\mathbf{Q})\right], \\
\left|M_{t}(\mathbf{Q})\right|^{2}=\frac{\hbar\left(e h_{14}\right)^{2}}{2 \varrho c_{t} Q} A_{t}(\mathbf{Q}),
\end{gathered}
$$

where $\varrho$ is the mass density of the crystal, $D$ is the deformation potential, $e h_{14}$ is the piezoelectric constant, $c_{\lambda}$ are sound velocities for longitudinal and transverse phonons, and $A_{\lambda}$ are the anisotropy factors, ${ }^{28}$

$$
\begin{gathered}
A_{l}(\mathbf{Q})=\frac{9 q^{4} Q_{z}^{2}}{2 Q^{6}}, \\
A_{t}(\mathbf{Q})=\frac{8 q^{2} Q_{z}^{4}+q^{6}}{4 Q^{6}} .
\end{gathered}
$$

\section{B. Approximate analytic form of $\mathcal{D}(\mathbf{q}, \boldsymbol{\omega})$}

While it is possible to obtain exact expressions for $\mathcal{D}$ from Eq. (14) including the full anisotropy functions [Eqs. (25a) and (25b)] and the form factors for infinite square wells, these are extremely complicated. Therefore, we shall make some well-controlled approximations explained below which do not significantly affect the final results for the computed transresistivity.

As we shall see shortly, the phonon-mediated effective interactions are important only when $\omega$ is close to $c_{\lambda} q$. For these $\omega$ 's the integral over $Q_{z}$ in Eq. (14) is dominated by contributions from near $Q_{z}=0$. We therefore remove all
$Q_{z}$-dependent factors, except for the small energy denominator, from the integral which defines $\mathcal{D}$. For example, one can set $Q_{z}=0$ in the anisotropy factors given in Eqs. (25a) and (25b), yielding

$$
A_{l} \approx 0, \quad A_{t} \approx \frac{1}{4} .
$$

Then the phonon-mediated effective interaction for two equivalent infinite square wells with width $L$ and center-tocenter separation of $d$ is

$$
\begin{aligned}
\nu_{0} \mathcal{D}_{i j}(q, \omega) \approx & -\frac{3 C_{\mathrm{DP}}}{k_{F} L} \delta_{i j} \\
& -C_{\mathrm{DP}} \frac{q \zeta_{l}^{2}}{k_{F} \sqrt{1-\zeta_{l}^{2}}} B_{i j}\left(q d \sqrt{1-\zeta_{l}^{2}}, q L \sqrt{1-\zeta_{l}^{2}}\right) \\
& -C_{\mathrm{PE}} \frac{k_{F}}{q \sqrt{1-\zeta_{t}^{2}}} B_{i j}\left(q d \sqrt{1-\zeta_{t}^{2}}, q L \sqrt{1-\zeta_{t}^{2}}\right) .
\end{aligned}
$$

Here, $\nu_{0}=m^{*} / \pi \hbar^{2}$, the two-dimensional electron-gas density of states (so that $\nu_{0} \mathcal{D}$ is dimensionless),

$$
\begin{gathered}
\zeta_{\lambda}=\frac{\omega}{c_{\lambda} q}+\frac{i}{2 q \ell_{\mathrm{ph}}}, \\
C_{\mathrm{PE}}=\frac{\left(e h_{14}\right)^{2} m^{*}}{8 \pi \hbar^{2} c_{t}^{2} \varrho k_{F}}, \\
C_{\mathrm{DP}}=\frac{D^{2} m^{*} k_{F}}{2 \pi \hbar^{2} c_{l}^{2} \varrho},
\end{gathered}
$$

and

$$
B_{i j}(x, y)=\left\{\begin{array}{l}
\frac{\pi^{2}}{y^{2}+\pi^{2}}\left(\frac{3 y}{2 \pi^{2}}+\frac{1}{y}+\frac{1}{2 y^{2}} \frac{\pi^{2}}{y^{2}+\pi^{2}}\left[e^{-2 y}-1\right]\right), \quad i=j \\
\exp (-x)\left(\frac{\pi^{2}}{y^{2}+\pi^{2}}\right)^{2} \frac{\sinh ^{2}(y)}{y^{2}}, \quad i \neq j .
\end{array}\right.
$$

In the expressions above, the square root with a positive real part should be taken.

Inserting numerical values for GaAs $\left(m^{*}=0.067 m_{e}, c_{l}\right.$ $=5.14 \times 10^{5} \mathrm{~cm} / \mathrm{s}, c_{t}=3.04 \times 10^{5} \mathrm{~cm} / \mathrm{s}, \varrho=5.3 \mathrm{~g} / \mathrm{cm}^{3}, e h_{14}$ $=1.2 \times 10^{7} \mathrm{eV} / \mathrm{cm}$, and $D=-13.0 \mathrm{eV}$ ), gives the following dimensionless coupling constants:

$$
\begin{gathered}
C_{\mathrm{PE}} \approx 1.64 \times 10^{-3} \times \frac{10^{6} \mathrm{~cm}^{-1}}{k_{F}}, \\
C_{\mathrm{DP}} \approx 2.7 \times 10^{-3} \times \frac{k_{F}}{10^{6} \mathrm{~cm}^{-1}} .
\end{gathered}
$$

For the same subband wave functions, the bare interlayer and intralayer Coulomb interactions are given by

$$
\nu_{0} U_{i j}(q)=\frac{q_{\mathrm{TF}}}{q} B_{i j}(q d, q L),
$$

where $q_{\mathrm{TF}}=2 \pi e^{2} \nu_{0}$ is the Thomas-Fermi wave vector. (We have absorbed the bulk dielectric constant of the semiconductor in the electron charge.)

\section{Strength of $\mathcal{D}_{21}$}

For the RPA screened Coulomb interaction $\left(U_{\mathrm{RPA}}\right)$ in a single-layer system, the magnitude of $\nu_{0} U_{\mathrm{RPA}}$ approaches 1 at long wavelengths. Although the corresponding value for double-layer systems is smaller, ${ }^{3}$ it is useful to compare $\nu_{0} \mathcal{D}_{21}$ to this value. For our present illustrative purpose we concentrate on the deformation potential term [i.e., the $D^{2}$ term in Eq. (23)], which turns out to dominate except for 
very low-density electronic systems. Then, in the limit of vanishing quantum well widths and $\ell_{\mathrm{ph}} \rightarrow \infty$, we obtain

$$
\begin{aligned}
\nu_{0} \mathcal{D}_{21}(q, \omega+i \eta) \approx & -C_{\mathrm{DP}} \frac{\omega^{2}}{q k_{F} c_{l}^{2} \sqrt{1-\omega^{2} /\left(q c_{l}\right)^{2}}} \\
& \times \exp \left(-d \sqrt{q^{2}-\omega^{2} c_{l}^{-2}}\right) .
\end{aligned}
$$

Note that the magnitude of the effective interaction diverges as $q \rightarrow \omega / c_{l}$ from above or below. [We point out that the transresistivity is strongly dependent on the width $L$, and therefore one should not take the $L \rightarrow 0$ limit when computing $\mathcal{D}$. We use the full form given by Eq. (27) in subsequent numerical calculations.]

The small prefactor $C_{\mathrm{DP}}$ guarantees that the phononmediated effective interaction is small compared to the Coulomb interaction except near $\omega \approx q c_{\lambda}$. The large value of the interaction in this region of phase space reflects the large phase space for intermediate states with small or vanishing energy denominators when $\omega \approx q c_{\lambda}$. (Note that the phonon energy varies slowly with $Q_{z}$ for $Q_{z}$ near zero.)

The importance for drag of the sharp peak in $\mathcal{D}_{21}(\mathbf{q}, \omega)$ is enhanced by the fact that it appears squared in Eq. (19). As a result, phonons do play an important role in frictional drag, even though the typical value of $\nu_{0} \mathcal{D}_{21}(\mathbf{q}, \omega)$ is small. In fact, if we ignore the effects of screening and let $\ell_{\mathrm{ph}} \rightarrow \infty$, we obtain an infinite transresistivity. This is easily seen from Eq. (35). The absolute value $\mathcal{D}_{21}$ diverges like $\left(c_{\lambda} q-\omega\right)^{-1 / 2}$ as $\omega$ approaches $c_{\lambda} q$ from both above and below. Inserting this bare form of the interaction into Eq. (19) gives a $\mid c_{\lambda} q$ $-\left.\omega\right|^{-1}$ divergence in the energy-transfer integral for every $q$, yielding an infinite transresistivity. This point does not seem to have been emphasized in the existing theoretical literature on this subject. As we show in the next two subsections, including either a finite mean free path or screening the interaction dynamically removes this spurious divergence.

In Fig. 2 we plot $\nu_{0}\left|\mathcal{D}_{21}\left(2 k_{F}, \omega\right) / \epsilon\left(2 k_{F}, \omega\right)\right|$ near the longitudinal resonance for different values of $\ell_{\mathrm{ph}}$. It is useful to compare $\nu_{0}\left|\mathcal{D}_{21} / \epsilon\right|$ to the screened interlayer Coulomb interaction $\nu_{0} U_{21, \mathrm{RPA}} \propto \exp (-q d) /\left(1+q_{\mathrm{TF}} / q\right)$. In contrast to the phonon-mediated interaction near the resonance, $\nu_{0} U_{21, \mathrm{RPA}}$ decreases rapidly below unity as a function of well separation. Figure 2 partly explains how the "weak" phonon-mediated interaction can compete with the Coulomb interaction as a mechanism for drag.

\section{Effect of screening}

From Eq. (27), one sees that the presence of a finite $\ell_{\text {ph }}$ cuts off the divergence and leads to a finite transresistivity. Since the divergence in the integrand is of the form $1 / \mid \omega$ $-c_{\lambda} q$, it follows that $\rho_{21}$ would be proportional to $\ln \left(\ell_{\mathrm{ph}}\right)$ if screening were not important. When screening is included, the drag resistivity does not diverge.

As shown in Eqs. (21) and (20), screening is accounted for by dividing the bare interlayer interaction $\mathcal{D}_{21}(q, \omega)$ by a dielectric function $\epsilon(q, \omega)$. Whenever $\mathcal{D}_{21}(q, \omega)$ diverges at $\omega=c_{\lambda} q$, so does $\epsilon(q, \omega)$. The screened interaction is therefore nondivergent, even when $\ell_{\mathrm{ph}} \rightarrow \infty$.

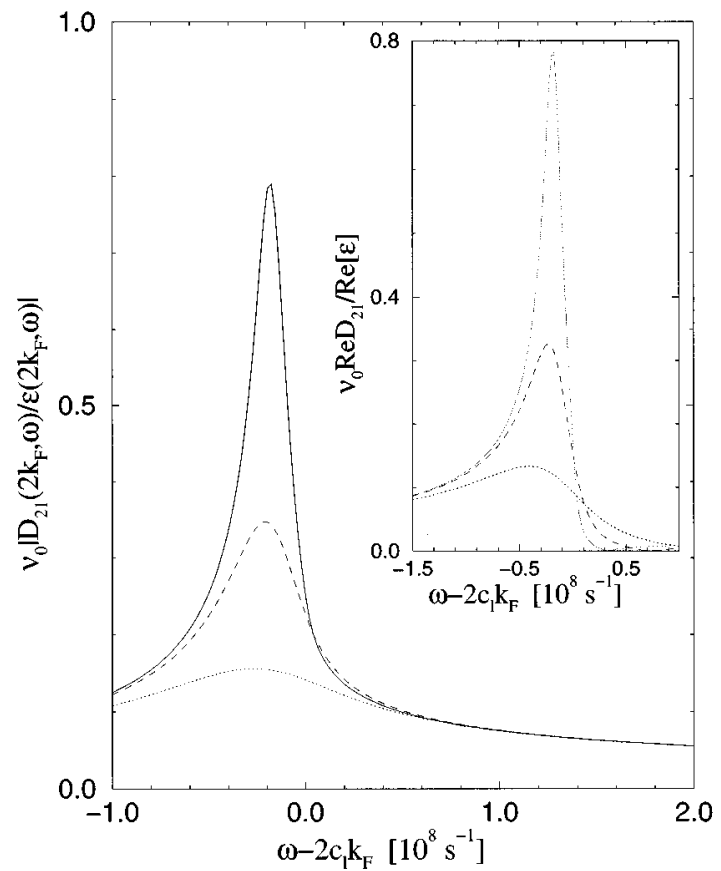

FIG. 2. The two-dimensional electron density of states times the screened phonon-mediated interaction $\nu_{0}\left|\mathcal{D}_{21}\left(2 k_{F}, \omega\right) / \epsilon\left(2 k_{F}, \omega\right)\right|$, as a function of frequency near the longitudinal resonance $\omega$ $\sim 2 c_{l} k_{F}$. The dotted, dashed, and solid lines are for $\ell_{\mathrm{ph}}$ $=0.1 \mathrm{~mm}, 0.3 \mathrm{~mm}$, and $1 \mathrm{~mm}$, respectively. Other parameters are $d=500 \AA$ and $L=200 \AA$, and the density $n=1.5 \times 10^{11} \mathrm{~cm}^{-2}$. The coupled electron-phonon mode occurs when $\nu_{0} \operatorname{Re} \mathcal{D}_{21} / \operatorname{Re}[\epsilon] \leqslant$ $-\frac{1}{2}$ [see Eq. (37)]. The inset indicates that for the given parameters the coupled electron-phonon mode develops at $q=2 k_{F}$ when $\ell_{\mathrm{ph}}$ $\gtrsim 0.5 \mathrm{~mm}$.

As we explain below, screening becomes important for the phonon exchange drag only if the phonon mean free path exceeds a critical value $\ell_{\text {ph,crit }}$. Since $\ell_{\text {ph,crit }}$ is close to realistic values, we investigate the two regimes separately in the following two sections.

\section{LONG MEAN FREE PATH LIMIT}

In this section we focus on the large phonon mean free path limit, where a coupled electron-phonon mode turns out to be of utmost importance. First we discuss the ideal case of infinite $\ell_{\mathrm{ph}}$, where the coupled mode is broadened by the coupling to the electronic system only. In this case we find an analytic form for the coupled mode contribution. Second, in Sec. IV B, we discuss how the collective mode contribution is modified by a finite mean free path and the conditions for its experimental observation.

\section{A. Infinite phonon mean free path limit}

The approximate analytic results discussed below apply only for layer separations smaller than a large but finite maximum value which we specify below; for still larger layer separations the analytic analysis is less revealing, and we have relied more strongly on numerical studies.

We show below that for $\ell_{\mathrm{ph}} \rightarrow \infty$, the real part of $\epsilon(q, \omega)$ vanishes, and the imaginary part is small enough to yield a 
sharp collective mode for $\omega$ just below $q c_{l}$ which contributes strongly to the frictional drag between the two layers. To make the following discussion as transparent as possible, we limit our attention to the case of identical electron layers so that $\mathcal{D}_{22}=\mathcal{D}_{11}$ and $U_{11}=U_{22}$. We locate the collective mode frequency $\omega_{0}(q)$ by solving the equation $\operatorname{Re}\left[\epsilon\left(q, \omega_{0}\right)\right]=0$. We will be interested in momentum transfers near $2 k_{F}$ which make the main contribution to the drag and systems with $k_{F} d \gg 1$ so that we can neglect $U_{21}(q) \propto \exp \left(-2 k_{F} d\right)$. The small layer separation approximation mentioned above consists of setting $\mathcal{D}_{11} \approx \mathcal{D}_{12} \equiv \mathcal{D}$ in Eq. (21), which is justified for

$$
d \ll \frac{1+q_{\mathrm{TF}} / 2 k_{F}}{16 C_{\mathrm{DP}} k_{F}} \equiv d_{B},
$$

as we show later. With these assumptions

$$
W_{21}(q, \omega) \approx \frac{\mathcal{D}_{21}(q, \omega)}{\left(1-U_{11} \chi\right)\left(1-U_{11} \chi-2 \mathcal{D} \chi\right)} .
$$

The collective mode in which we are interested occurs near $\omega=c_{l} q \ll v_{F} q$, and hence is in the low-frequency regime for $\chi(q, \omega)$. Here $v_{F}$ is the Fermi velocity of the electronic system. We therefore approximate $\operatorname{Re} \chi(q, \omega) \approx \chi(q, \omega=0)=$ $-\nu_{0}$. We write the imaginary part of the polarization function in the form $\operatorname{Im} \chi(q, \omega)=-\nu_{0} \widetilde{\delta}$. In the low-frequency limit of the RPA, $\widetilde{\delta}\left(q, c_{l} q\right) \approx c_{l} /\left(v_{F} \sqrt{1-q^{2} / 4 k_{F}^{2}}\right)$, except near $2 k_{F}$, where it is approximately $\sqrt{c_{l} / 2 v_{F}}$. $\widetilde{\delta}$ is dimensionless and has a wave vector and frequency dependence which will be left implicit in the following discussion except where emphasis is important. However, it is important for the following discussion that $\widetilde{\delta}\left(q, c_{l} q\right)$ is generally small compared to 1 .

Because we are interested in large wave vectors near $q$ $=2 k_{F}$ we include only longitudinal deformation potential coupling. The calculation including transverse piezoelectric mode follows mutatis mutandis. From Sec. III we have, for $\omega$ close to but smaller than $c_{l} q$,

$$
\nu_{0} W_{21}(q, \omega)=\frac{-C_{\mathrm{DP}}}{\epsilon_{0}\left(\frac{k_{F}}{q} \epsilon_{0} \sqrt{1-\omega^{2} / c_{l}^{2} q^{2}}-2 C_{\mathrm{DP}}\right)-i 2 C_{\mathrm{DP}} \widetilde{\delta}} .
$$

In this equation $\epsilon_{0}=1+q_{\mathrm{TF}} / q$ is the RPA static dielectric function for an isolated layer. The collective mode occurs where the real part of denominator of Eq. (38) vanishes at

$$
\omega_{0}=c_{l} q \sqrt{1-\left(\frac{2 q C_{\mathrm{DP}}}{k_{F} \epsilon_{0}}\right)^{2}} .
$$

This collective mode results from coupling of the electron layers to phonons with $\omega$ close to $c_{l} q$. Expanding the denominator of Eq. (38) around the pole, we find that for $\omega$ close to $\omega_{0}$,

$$
\left|\nu_{0} W_{21}(q, \omega)\right|^{2}=\frac{c_{l} q^{3} C_{\mathrm{DP}}^{2}}{\epsilon_{0}^{3} k_{F}^{2} \widetilde{\delta}} \frac{\Gamma}{\left(\omega-\omega_{0}\right)^{2}+\Gamma^{2}},
$$

where the width of the collective mode resonance is given by

$$
\Gamma=\frac{4 \widetilde{\delta} c_{l} q^{3} C_{\mathrm{DP}}^{2}}{\epsilon_{0}^{3} k_{F}^{2}} .
$$

Note that the width of the resonance is small compared to the shift of the resonance from $c_{l} q$ and that the resonance line shape is approximately Lorentzian only if $\widetilde{\delta} \ll 1$. Where this condition is not satisfied, Eq. (40) will not be accurate.

Numerical calculations discussed below demonstrate that for $\ell_{\mathrm{ph}} \rightarrow \infty$ the drag is dominated by coupling associated with this collective mode resonance. Since the effective interaction has a more rapid frequency variation than other quantities in the expression for the transresistivity [Eq. (19)], we may approximate the screened interaction near the resonance by a $\delta$ function:

$$
\left|\nu_{0} W_{21}(q, \omega)\right|^{2}=\frac{c_{l} q^{3} C_{\mathrm{DP}}^{2}}{k_{F}^{2} \epsilon_{0}^{3} \widetilde{\delta}} \pi \delta\left(\omega-\omega_{0}\right) .
$$

This approximation allows the frequency integral in Eq. (19) to be performed, and the contribution from the coupled mode to the transresistivity may be expressed as

$$
\rho_{21, \lambda} \approx\left(\frac{h}{e^{2}}\right) \frac{\pi K_{\lambda}}{n^{2} k_{B} T} \int_{0}^{\infty} d q\left(\frac{q^{\alpha}}{\epsilon_{0}^{3}}\right) \frac{\nu_{0} \operatorname{Im} \chi\left(q, c_{\lambda} q\right)}{\sinh ^{2}\left(\hbar c_{\lambda} q / 2 k_{B} T\right)},
$$

where $\alpha=6$ for the longitudinal phonons and $\alpha=2$ for the transverse phonons, and where

$$
\begin{aligned}
& K_{l}=\frac{\hbar D^{4}}{(4 \pi)^{3} c_{l}^{3} \varrho^{2}}, \\
& K_{t}=\frac{\hbar\left(e h_{14}\right)^{4}}{2^{10} \pi^{3} c_{t}^{3} \varrho^{2}} .
\end{aligned}
$$

The $q^{6} / \epsilon_{0}^{3}$ term in the integrand above implies that for $T$ $\geq T_{\mathrm{BG}}=2 \hbar c_{l} k_{F} / k_{B}$, the main contribution to the integral comes from the $q=2 k_{F}$ region; i.e., large-angle scattering dominates the phonon-mediated drag.

Comparing with Eq. (2) we see that, at least for $\ell_{\mathrm{ph}} \rightarrow \infty$, the phonon-mediated drag can be comparable to or stronger than Coulomb drag, in spite of the weak electron-phonon interactions. Crudely the condition which needs to be satisfied is that $T_{\mathrm{BG}}$ is low enough or the layer separation is large enough that $C_{\mathrm{DP}}^{2} \sqrt{c_{l} / v_{F}}$ $\geq\left(k_{B} T_{\mathrm{BG}} / \epsilon_{F}\right)^{2}\left(q_{\mathrm{TF}} d\right)^{-2}\left(k_{F} d\right)^{-2}$. For typical layer densities in $\mathrm{GaAs}$, this condition is satisfied for layer separations larger than a few tens of nanometers, consistent with experimental observations. When phonon-mediated drag is dominant, $\rho_{21}$ will be proportional to temperature for $T \gg T_{\mathrm{BG}}$. Below $T_{\mathrm{BG}}$ there will be a crossover to a regime where the piezoelectric contribution dominates and the temperature dependence goes approximately as $T^{5}$. This can be seen from Eq. (43) by defining an integration variable proportional to $q / T$, and taking the $q$ dependence of $\epsilon_{0}$ into account. We shall discuss this further in Sec. VI A. At extremely low temperatures, the assumption that the drag rate is determined by the resonance $\omega \simeq c_{l} q$ will break down. $\rho_{21}$ is then dominated by the $\omega=0$ limit of $\mathcal{D}_{21}$ and will revert to the familiar $T^{2}$ law for carrier-carrier scattering in a Fermi liquid. 
Recall that we used $\mathcal{D}_{21} \approx \mathcal{D}_{11}$ to obtain the above results. From Eq. (27),

$$
\frac{\mathcal{D}_{21}^{2}\left[q, \omega_{0, l}(q)\right]}{\mathcal{D}_{11}^{2}\left[q, \omega_{0, l}(q)\right]} \approx \exp \left[-2 q d \sqrt{2\left(1-\omega_{0} / c_{l} q\right)}\right]
$$

Hence, for the approximation $\mathcal{D}_{11}^{2}-\mathcal{D}_{21}^{2} \approx 0$ to be valid, one must have $2 q d \sqrt{2\left(1-\omega_{0} / c_{l} q\right)} \ll 1$. Together with Eq. (39), this gives the condition Eq. (36). For $\ell_{\mathrm{ph}} \rightarrow \infty$, the preceding approximate calculation implies that there is no layer separation dependence until this length, which for GaAs and typical densities corresponds to $d \sim 5000 \AA$, is reached. Numerical results show, however, that there is a weak distance dependence on the transresistivity in this large- $\ell$ regime. This stems from the presence of a relatively long nonLorentzian tail for $\omega<c_{l} q$ which contributes significantly to the integral, as we will discuss below.

At larger layer separations, the interlayer phonon propagator $\mathcal{D}_{21}$ is reduced at the collective-mode frequency and the two electron layers interact with the phonon system more independently. We must then use a more refined expression for the effective interaction:

$$
W_{21}(q, \omega) \approx \frac{\mathcal{D}_{21}(q, \omega)}{\left(1-\left[U_{11}+\mathcal{D}_{11}\right] \chi\right)^{2}-\mathcal{D}_{21}^{2} \chi^{2}} .
$$

A competition occurs between a decline in the coupling due to suppression of the interlayer propagator $\mathcal{D}_{21}$ and resonant enhancement of the electron-phonon interaction near each electron layer.

\section{B. Coupled electron-phonon mode with finite mean free path}

The above analysis is based on phonons with an infinite mean free path, apart from the finite lifetimes due to interactions with the electronic layers. Any real system will have imperfections which will make the phonon lifetime finite even when the electron layers are not present. Even when the lattice is perfect and free of isotopic impurities, anharmonicity and boundary scattering will cause phonon modes to decay. In the following we represent all these effects in the simplest possible way by assigning a common phenomenological mean free path $\ell_{\mathrm{ph}}$ to all modes.

The occurrence of coupled phonon-plasmon collective modes, signaled by by a zero in the $\operatorname{Re}[\epsilon(q, \omega)]$, requires a cancellation between phonon-mediated and Coulomb interaction contributions. In the present section we will consider the limit in which the phonon-mediated contributions to $\epsilon(q, \omega)$ can be neglected, i.e., the limit in which $\left|\nu_{0} \mathcal{D}\right| \ll 1$ for almost all energy and momentum transfers and for both intralayer and interlayer propagation. We will see that this condition is satisfied except at long phonon mean free paths.

For $\omega$ close to $q c_{l}$ and finite $\ell_{\mathrm{ph}}$ (but large compared to $\left.q^{-1}\right)$

$$
\left|\nu_{0} \mathcal{D}_{21}\right| \approx\left(q C_{D P} / k_{F}\right)\left|\sqrt{1-\zeta_{l}^{2}}\right|^{-1}
$$

where $\zeta_{l}$ is defined in Eq. (28), and $1-\zeta_{l}^{2} \approx 1-\left(\omega / q c_{l}\right)^{2}$ $+i / q \ell_{\mathrm{ph}}$. There is a critical value $\ell_{\mathrm{ph}, \text { crit }}$ such that 1 $-\left(\omega_{0} / c_{l} q\right)^{2} \lesssim 1 / q \ell_{\text {ph,crit }}$, in which case $\left|\operatorname{Re}\left[\mathcal{D}_{i j}\right]\right|$ never be- comes large enough for $\operatorname{Re}[\epsilon]=0$ to have a solution, and the collective mode ceases to exist. This critical value is given by

$$
\ell_{\mathrm{ph}, \mathrm{crit}}=\frac{\epsilon_{0}^{2} k_{F}^{2}}{4 C_{\mathrm{DP}}^{2} q^{3}}=\frac{\left(1+q_{\mathrm{TF}} / 2 k_{F}\right)^{2}}{32 C_{\mathrm{DP}}^{2} k_{F}},
$$

where, since large momentum transfers dominate the drag, we have set $q=2 k_{F}$. $\ell_{\text {ph,crit }}$ is even longer at smaller $q$ 's, and it seems improbable that the collective mode discussed in Sec. III would ever be evident in inelastic light scattering studies of long-wavelength electronic excitations. In GaAs, and for $k_{F} \approx 10^{6} \mathrm{~cm}^{-1}$, the critical mean free path is approximately $\ell_{\text {ph,crit }} \approx 0.2 \mathrm{~mm}$. The actual mean free path of course depends on the sample in question.

It is often the case that scattering of phonons off the boundaries of the sample can be accounted for by taking the mean free path in the absence of bulk scatterers to be equal to the sample size. We argue below that for the present problem, the sample size in the $z$ direction is usually irrelevant, and that the maximum mean free path is given by the typically larger lateral dimension of the sample.

The phonons which contribute to the coupled mode are those which are confined around the two-dimensional electron gases (2DEG's). The extent of the confinement is given by range $\mathcal{D}_{21}$ in the $z$ direction (i.e., perpendicular to the 2DEG's). To study the $z$-direction range of the phonon field participating in the collective mode, we look more closely at which $z$ region actually contributes to the coupling. We write the effective phonon-mediated interaction given in Eq. (14) as

$$
\begin{aligned}
D_{21}(q, \omega+i \delta)= & \int d z d z^{\prime}\left|\varphi_{2}(z)\right|^{2}\left|\varphi_{1}\left(z^{\prime}\right)\right|^{2} \\
& \times K\left(q, \omega+i \delta, z-z^{\prime}\right),
\end{aligned}
$$

where $K\left(q, \omega+i \delta, z-z^{\prime}\right)$ is the Fourier transform of the phonon Green's function and the electron-phonon coupling matrix element with respect to $Q_{z}$,

$$
K(q, \omega+i \delta, z)=\sum_{\lambda} \int \frac{d Q_{z}}{2 \pi \hbar} e^{i Q_{z} z} D_{\lambda}(\mathbf{Q}, \omega+i \delta)\left|M_{\lambda}(\mathbf{Q})\right|^{2} .
$$

For a given $q$ and $\omega$, the spatial extent in the $z$ direction of $K(q, \omega+i \delta, z)$ gives the range of the phonon field. Since the collective-mode frequencies are very close to $\omega=c_{l} q$, the relevant phonon wave vectors in the $z$ direction are small, and the same approximations used previously can also be applied here, yielding an approximate form for $K$ given by Eq. (35) with $d$ replaced by $\left|z-z^{\prime}\right|$, i.e., $K\left(q, \omega+\delta, z-z^{\prime}\right)$ $\sim \exp \left(-\left|z-z^{\prime}\right| \sqrt{q^{2}-\omega^{2} / c_{l}^{2}}\right)$. The spatial extent of the phonon field participating in the collective mode can be found by substituting the frequency for mode $\omega_{0}$ into this exponential form, giving $K \sim \exp \left(-\left|z-z^{\prime}\right| / d_{B}\right)$, where $d_{B}$ was defined previously in Eq. (36). For GaAs at $n=1.5 \times 10^{11} \mathrm{~cm}^{-2}, d_{B}$ is approximately $5000 \AA$. Any boundaries or imperfections beyond this range in the $z$ direction have a negligible effect.

It is plausible that some existing experimental results are for samples with $\ell_{\mathrm{ph}}$ in the lateral direction comparable to this critical mean free path, and hence partially reflect col- 
lective excitations of the electron-phonon system. However, more experiments are needed before definite conclusions can be drawn.

\section{SHORT PHONON MEAN FREE PATH LIMIT}

In the present section, we will consider the limit in which phonon-mediated contributions to $\epsilon(q, \omega)$ can be neglected, i.e., $\ell_{\mathrm{ph}} \ll \ell_{\text {ph,crit }}$. We begin our discussion by addressing the distinction between the real and virtual phonon contributions. This we do partly due to somewhat confusing use of these terms in the existing literature.

\section{A. Real and virtual phonons}

The phonon-mediated effective electron-electron interaction $\mathcal{D}_{21}(q, \omega)$, obtained here from diagrammatic perturbation theory, can also be derived (with a little more work) using elementary time-dependent perturbation theory starting from an expression of the form ${ }^{29}$

$$
\mathcal{D}_{21}(q, \omega)=\sum_{I} \frac{\left\langle i\left|\hat{H}_{e-\mathrm{ph}}\right| I\right\rangle\left\langle I\left|\hat{H}_{e-\mathrm{ph}}\right| f\right\rangle}{E_{i}-E_{I}+i \eta} .
$$

Here $|i\rangle,|f\rangle$, and $|I\rangle$ are the initial state, the final state in which momentum has been transferred between the layers, and an intermediate state in which the momentum to be transferred is carried by a nonequilibrium phonon. The infinitesimal imaginary part $i \eta$ in the denominator enforces causality, ${ }^{29}$ and plays a crucial role when intermediate and initial or final states are close in energy. In time-dependent perturbation theory, this form of effective interaction determines the transition rate between initial and final states when the perturbing term in the Hamiltonian, the electron-phonon interaction in the present case, does not directly couple initial and final states. We therefore expect the phonon-mediated effective interaction to play the same role in transport experiments as the interlayer Coulombic interaction which does have direct matrix elements between initial and final states. The intermediate state need not conserve energy, and, in the jargon of time-dependent perturbation theory, is consequently referred to as a virtual state.

The intermediate states are ones where a phonon of momentum $\mathbf{Q}=\left(\mathbf{q}, Q_{z}\right)$ has been created or destroyed by an electron in layer 1 or 2 . Summing over four intermediate states for each $Q_{z}$, and performing thermal averages, gives the following expression for transition matrix elements with 2D wave-vector transfer $\mathbf{q}$ :

$$
\begin{aligned}
\mathcal{D}_{21}(q, \omega)= & \int \frac{d Q_{z}}{2 \pi}\left|M_{l}(Q)\right|^{2} F_{1}\left(Q_{z}\right) F_{2}\left(-Q_{z}\right)\left[\frac{1}{\omega-\omega_{\mathbf{Q}}+i \eta}\right. \\
& \left.-\frac{1}{\omega+\omega_{\mathbf{Q}}-i \eta}\right] .
\end{aligned}
$$

Equation (14) reduces to this form for $\ell_{\mathrm{ph}} \rightarrow \infty$, except that we have, for simplicity, retained only the longitudinal phonons in the present discussion.
Recalling that $\hbar \omega$ is the energy transferred between the layers, and $\hbar \omega_{\mathbf{Q}}$ is the energy of the intermediate phonon, when the denominator vanishes in Eq. (52) energy is conserved in the intermediate state. Therefore, the real (imaginary) part of the term in the square parentheses gives the virtual (real) phonon contribution. In analogy, we define the virtual-phonon exchange contribution to the effective interaction as the contributions from $\operatorname{Re} D$ in Eq. (14), and the real-phonon exchange contribution as that from $\operatorname{Im} D$. Ignoring the anisotropy factors in the phonon matrix elements for the deformation potential, it turns out that in the $\ell_{\mathrm{ph}} \rightarrow \infty$ limit, the entire $\omega<c_{l} q$ contribution to $\mathcal{D}_{21}$ is "virtual" and the entire $\omega>c_{l} q$ contribution is "real." In general, the division between the contributions are not so clearcut; at a given $\omega$ and $q$ both virtual and real contributions could exist simultaneously.

In a semiclassical transport theory, real-phonon processes result in a nonequilibrium distribution of phonons in a coupled electron-phonon Boltzmann equation. In Appendix A we derive an expression for the real-phonon exchange contribution to the drag resistance using such a coupled Boltzmann equation approach, and explicitly demonstrate its equivalence to the purely real-phonon contribution of $\mathcal{D}_{21}$ to the drag. The appearance here of both virtual- and realphonon contributions to the transresistivity in a single Feynman diagram is reminiscent of the appearance of both contributions to the quasiparticle scattering rate re-32 $^{30}$ in the phonon exchange contribution to the electron self-energy of a 3D electron-phonon system.

\section{B. Reciprocal-space calculation}

In the following subsections we elucidate the physics of the drag for short phonon mean free paths. When $\ell_{\mathrm{ph}}$ $\ll \ell$ ph,crit, we can set the $\mathcal{D}_{i j}$ factors in the expression $\epsilon$ to zero. The screened interlayer interaction is then simply

$$
W_{21}(q, \omega) \simeq \frac{\mathcal{D}_{21}(q, \omega)}{\left(1-U_{11}(q) \chi(q, \omega)\right)^{2}} \simeq \frac{\mathcal{D}_{21}(q, \omega)}{\epsilon_{0}^{2}}
$$

where we have taken the static limit of $\chi$ appropriate for temperatures comparable to $T_{\mathrm{BG}}$. The transresistivity is then given by

$$
\begin{aligned}
\rho_{21, l} \simeq & \frac{-\hbar^{2}}{8 \pi^{2} e^{2} n_{1} n_{2} k_{B} T} \\
& \times \int_{0}^{\infty} d q q^{3} \frac{\operatorname{Im} \chi_{1}\left(q, c_{l} q\right) \operatorname{Im} \chi_{2}\left(q, c_{l} q\right)}{\sinh ^{2}\left(\hbar c_{l} q / 2 k_{B} T\right)} \frac{1}{\nu_{0}^{2} \epsilon_{0}^{4}} \\
& \times \int_{0}^{\infty} d \omega\left|\nu_{0} \mathcal{D}_{21}(q, \omega)\right|^{2} .
\end{aligned}
$$

We have factored terms which vary slowly with respect to $\omega$ out of the $\omega$ integration, since $\mathcal{D}_{21}(q, \omega)$ is relatively sharply peaked around $\omega=c_{l} q$.

From Eqs. (27) and (31), $\nu_{0} \mathcal{D}_{12}$ for $\omega \approx c_{l} q$ is given approximately by 


$$
\nu_{0} \mathcal{D}_{21}(q, \omega) \simeq \begin{cases}-q^{2} C_{\mathrm{DP}} f_{\mathrm{cut}}(\tilde{q}) \exp (-d \widetilde{q}) /\left(k_{F} \widetilde{q}\right), & q-\omega / c_{l} \gg \ell_{\mathrm{ph}}^{-1} \\ q^{2} C_{\mathrm{DP}} f_{\mathrm{cut}}(\widetilde{q}) \exp \left(i d \tilde{q}-\omega d / 2 c_{l} \ell \ell_{\mathrm{ph}} \widetilde{q}\right) /\left(i k_{F} \widetilde{q}\right), & \omega / c_{l}-q \gg \ell_{\mathrm{ph}}^{-1},\end{cases}
$$

where $\tilde{q}=\left|q^{2}-\left(\omega / c_{l}\right)^{2}\right|^{1 / 2}$, and $f_{\text {cut }}(\widetilde{q})$ is a function (dependent on the form factor) which cuts off at $\tilde{q} \sim L^{-1}$.

In the $c_{l} q>\omega$ case, the effective interaction is dominated by virtual phonon exchange, whereas, in the $\omega>c_{l} q$ case, the integral is dominated by energy-conserving intermediate states and the effective interaction is due to real-phonon exchange. The analytic expression for the integral is complicated in the small region where $\left|\omega-c_{l} q\right|<c_{l} / \ell_{\mathrm{ph}}$, and we ignore it in our treatment below. The smaller $\ell_{\mathrm{ph}}$ is, the wider this region becomes, and hence the expressions derived below are not quantitatively valid for small $\ell_{\mathrm{ph}}$ (below $10^{4} \mathrm{~nm}$ for typical parameters in GaAs); however, the expressions seem to exhibit the correct qualitative behavior when compared to numerical calculations even for small $\ell_{\mathrm{ph}}$.

Parameters of experimental systems studied to date satisfy the inequalities $\ell_{\mathrm{ph}} \gg d>L$ and $\left(2 k_{F} L\right)^{2} \gg 1$. It follows that for $\omega$ near $c_{l} q$ the integrand of the frequency integral in the drag resistivity expression is proportional to $\tilde{q}^{-2} \approx(2 q \mid q$ $\left.-\omega / c_{l} \mid\right)^{-1}$. The logarithmic divergence of the integral is cut off at small $\tilde{q}$ by the validity limits in Eq. (55), and at large $\tilde{q}$ by the exponential suppression factors or cutoff functions which appear in Eq. (55). For the real-phonon contribution we find

$$
\begin{aligned}
& \int_{q c_{l}}^{\infty} d \omega\left|\nu_{0} \mathcal{D}_{21}(q, \omega)\right|^{2} \\
& \simeq \frac{q^{3} C_{\mathrm{DP}}^{2} c_{l}}{k_{F}^{2}} \int_{\tilde{q}_{\text {min }}^{r}}^{\tilde{q}^{r}} d \tilde{q} \frac{\exp \left(-q d / \ell_{\mathrm{ph}} \tilde{q}\right)}{\tilde{q}} \\
& =\frac{q^{3} C_{\mathrm{DP}}^{2} c_{l}}{k_{F}^{2}}\left[\operatorname{Ei}\left(-d \sqrt{\frac{2 q}{\ell_{\mathrm{ph}}}}\right)-\mathrm{Ei}\left(-\frac{q d L}{\ell_{\mathrm{ph}}}\right)\right],
\end{aligned}
$$

where

$$
\begin{gathered}
\widetilde{q}_{\min }^{r}=\left(q / 2 \ell_{\mathrm{ph}}\right)^{1 / 2}, \\
\widetilde{q}_{\max }^{r}=L^{-1},
\end{gathered}
$$

and $\operatorname{Ei}(x) \equiv \int_{-\infty}^{x} d t \exp (t) / t$ is the exponential integral, which has the limiting behavior

$$
\operatorname{Ei}(-x) \sim\left\{\begin{array}{l}
\ln (x), \quad 0<x \ll 1 \\
-\exp (-x) / x, \quad x \gg 1
\end{array}\right.
$$

The upper cutoff $\widetilde{q}_{\max }$ reflects the fact that it is impossible to excite phonons with a $z$-wave vector larger than $L^{-1}$. The $\tilde{q}$ integration in Eq. (56) can by physically interpreted as follows. The velocity component of a phonon in the $z$ direction is approximately $c_{l} \widetilde{q} / q$, and hence the time taken to travel a distance $d$ in the $z$ direction is

$$
t_{\text {trans }}=q d / c_{l} \tilde{q}
$$

The exponential factor in Eq. (56) is $\exp \left(-t_{\text {trans }} c_{l} / \ell_{\mathrm{ph}}\right)$, which is the probability that a real phonon emitted from one layer reaches the other.

For the virtual-phonon contribution we find

$$
\begin{aligned}
\int_{0}^{q c_{l}} d \omega\left|\nu_{0} \mathcal{D}_{21}(q, \omega)\right|^{2} & \simeq \frac{q^{3} C_{\mathrm{DP}}^{2} c_{l}}{k_{F}^{2}} \int_{q_{\min }^{v}}^{\infty} d \tilde{q} \frac{\exp (-2 d \tilde{q})}{\tilde{q}} \\
& =-\frac{q^{3} C_{\mathrm{DP}}^{2} c_{l}}{k_{F}^{2}} \mathrm{Ei}\left(-d \sqrt{\frac{2 q}{\ell_{\mathrm{ph}}}}\right),
\end{aligned}
$$

where $q_{z, \min }^{v}=(q / 2 \ell \mathrm{ph})^{1 / 2}$ is also given by the validity limit in Eq. (55). The term in the exponent can be interpreted as the probability that a virtual phonon emitted by one layer reaches the other. The lifetime of the virtual state which is given by the energy-time uncertainty relation $\Delta t \sim\left(q / c_{l}\right.$ $-\omega)^{-1} \sim \widetilde{q}^{2} / c_{l} q$. The probability is $\exp \left(-t_{\text {trans }} / \Delta t\right)$, where the transit time is given in Eq. (59). This gives the exponential factor, and hence the distance dependence in Eq. (60).

Only the sum of the two contributions will be observed in experiments. Adding the two terms yields

$$
\int_{0}^{\infty} d \omega\left|\nu_{0} \mathcal{D}_{21}(q, \omega)\right|^{2} \approx-\frac{q^{3} c_{l} C_{\mathrm{DP}}^{2}}{k_{F}^{2}} \operatorname{Ei}\left(-\frac{q d L}{\ell_{\mathrm{ph}}}\right) \approx \frac{q^{3} c_{l} C_{\mathrm{DP}}^{2}}{k_{F}^{2}} \times\left\{\begin{array}{l}
\ln \left(\frac{\ell_{\mathrm{ph}}}{q L d}\right), q L d / \ell_{\mathrm{ph}} \ll 1 \\
\ell_{\mathrm{ph}} \exp \left(-q L d / \ell_{\mathrm{ph}}\right) /(q L d), \quad q L d / \ell_{\mathrm{ph}} \gg 1 .
\end{array}\right.
$$


Inserting Eq. (61) into Eq. (54), we obtain

$$
\begin{aligned}
\rho_{21} \approx & \left(\frac{h}{e^{2}}\right) K_{\lambda} \frac{\operatorname{Ei}\left(-2 k_{F} L d / \ell_{\mathrm{ph}}\right)}{n_{1} n_{2} k_{B} T} \\
& \times \int_{0}^{\infty} d q\left(\frac{q^{\alpha}}{\epsilon_{0}^{4}}\right) \frac{\operatorname{Im} \chi_{1}\left(q, c_{\lambda} q\right) \operatorname{Im} \chi_{2}\left(q, c_{\lambda} q\right)}{\sinh ^{2}\left(\hbar c_{\lambda} q / 2 k_{B} T\right)},
\end{aligned}
$$

where $K_{\lambda}$ was defined in Eq. (44a), and again $\alpha=6$ for $\lambda$ $=l$ and $\alpha=2$ for $\lambda=t$. The argument of the exponential integral was replaced by the typical value $\ell_{\mathrm{ph}} / 2 L k_{F} d$ since it varies slowly over the large-angle scattering region which dominates the integral $\left(k_{F}=\min \left[k_{F, 1}, k_{F, 2}\right]\right)$. Equation (62) gives low- $T$ power laws for $\rho_{21}$ of $T^{10}$ for $\lambda=l$ and $T^{6}$ for $\lambda=t$.

The distance dependence in the short phonon mean free path limit has now been made explicit; as $d$ is increased, the transresistivity falls logarithmically when $\ell_{\mathrm{ph}} / 2 L k_{F} d \gtrsim 1$, and exponentially when $\ell_{\mathrm{ph}} / 2 L k_{F} d \lesssim 1$. The dependence of the transresistivity upon temperature, electron density, and the ratio of the electron densities $n_{1} / n_{2}$ is given by the remaining integral over the wave vector, which essentially expresses the phase space available for large angle scattering, as we shall discuss in Sec. VI.

\section{Real-space calculation}

In many physics problems, translational invariance and time independence makes reciprocal-space calculations, like the one presented in Sec. V B simple and convenient. It is often the case, however, that the corresponding real-space calculations provide a useful alternate language for physical interpretation. In the present case the frequency integral in Eq. (54) is equivalent to an integral over time,

$$
\int_{-\infty}^{\infty} d \omega\left|\nu_{0} \mathcal{D}_{21}(q, \omega)\right|^{2}=2 \pi \int_{-\infty}^{\infty} d t\left|\nu_{0} \mathcal{D}_{21}(q, t)\right|^{2},
$$

where

$$
\nu_{0} \mathcal{D}_{21}(q, t) \simeq \frac{c_{l} q C_{\mathrm{DP}}}{2 \pi k_{F}} \int_{-\infty}^{\infty} d Q_{z} F_{1}\left(Q_{z}\right) F_{2}\left(-Q_{z}\right) D(\mathbf{Q}, t)
$$

and the real-time, retarded, phonon propagator is given by

$$
D(\mathbf{Q}, t)=-2 \Theta(t) \sin \left(\omega_{\mathbf{Q}} t\right) e^{-t / 2 \tau_{\mathrm{ph}}},
$$

where $\tau_{\mathrm{ph}}=\ell_{\mathrm{ph}} / c_{l}$. Since the important values of $Q_{z}$ are small compared to $q$, we can expand

$$
\omega_{\mathbf{Q}}=c_{l} \sqrt{q^{2}+Q_{z}^{2}} \approx c_{l} q+\frac{c_{l}}{2 q} Q_{z}^{2}
$$

Notice that the phonons have a quadratic dispersion in the $Q_{z}$ direction, but a linear dispersion in the plane of the electron layers. The $Q_{z}$ integration in Eq. (64) can now be performed if we model the density profiles as Gaussians, so that $F_{1}\left(Q_{z}\right) F_{2}\left(-Q_{z}\right)=e^{-L^{2} Q_{z}^{2}} e^{-i d Q_{z}}$. We find that

$$
\begin{aligned}
& \int_{-\infty}^{\infty} d \omega\left|\nu_{0} \mathcal{D}_{21}(q, \omega)\right|^{2} \exp \left(-\frac{1}{2} \frac{d^{2}}{L^{2}+\left(\frac{c_{l} t}{2 q L}\right)^{2}}\right) \\
&\left.\approx \frac{c_{l}^{2} q^{2} C_{\mathrm{DP}}^{2}}{L k_{F}^{2}} \int_{0}^{\infty} d t e^{-t / \tau_{\mathrm{ph}}} \frac{\left(\sqrt{c_{l} t}\right)^{2}}{2 q L}\right)^{2}
\end{aligned}
$$

This expression suggests a picture in real time for the phonon-mediated interaction. The integrand of the time integral is recognized as a wave packet which is centered around one well, and which broadens and decays as time evolves. When the wave packet is broad enough to reach the other well, a transfer of momentum (parallel to the layers) can occur. The distance dependence of the interaction is determined by the time dependence of the intensity of the phonon field disturbance at the other well. Since $d^{2} \gg L^{2}$ there will be no contributions to the integral in Eq. (67) until $t \simeq t^{*}$ $=q L d / c_{l}$ which corresponds to the time it takes the wave packet to reach the second well. For $t>t^{*}$ the square of the amplitude of the wave packet at the second well [the integrand in Eq. (67)] falls off as $1 / t$ until it is eventually cut off at $t \simeq \tau_{\mathrm{ph}}$. If $\tau_{\mathrm{ph}}<t^{*}$, then only the exponentially small tail of the phonon field impinges on the second well. Defining $s \equiv c_{l} t / q L d$ the time integral, Eq. (67) can be written

$$
\begin{aligned}
\int_{-\infty}^{\infty} d \omega\left|\nu_{0} \mathcal{D}_{21}(q, \omega)\right|^{2} \approx & \frac{2 C_{\mathrm{DP}}^{2} c_{l} q^{3}}{k_{F}^{2}} \int_{1}^{\infty} d s \frac{1}{s} \\
& \times \exp \left(-q L \frac{d}{\ell_{\mathrm{ph}}} s\right) \exp \left(-\frac{2}{s^{2}}\right) .
\end{aligned}
$$

This integral cannot be evaluated analytically, but as $s>1$ the term $\exp \left(-1 / 2 s^{2}\right)$ can be approximated by unity. The resulting integral then yields Eq. (61). Therefore, the $d$ dependence of $\rho_{21}$ can be interpreted as coming from the interplay of the layer separation and the range of the phonons, which mediate the drag.

In the regime of short phonon mean free paths, the drag is mediated by damped phonons, some of which spread over large distances in the $z$ direction. Hence in this regime the boundaries in the $z$ direction will limit the effective $\ell_{\mathrm{ph}}$. When boundary scattering dominates, a complete theory would require a realistic description of phonons scattering off the sample boundaries. This is in contrast to the limit $\ell_{\mathrm{ph}} \gg \ell_{\mathrm{ph}, \text { crit }}$, where the drag is dominated by a coupled mode which is localized on the length scale of $d_{B}$, and boundaries beyond this range are irrelevant.

\section{NUMERICAL RESULTS}

The results presented in this section were obtained by numerical evaluation of the transresistivity formula [Eq. (19)] using the complete expressions for both the effective interaction [Eq. (14)] and the screening function [Eq. (21)]. Although these numerical calculations are free of some of the approximations used in the preceding sections in order to 


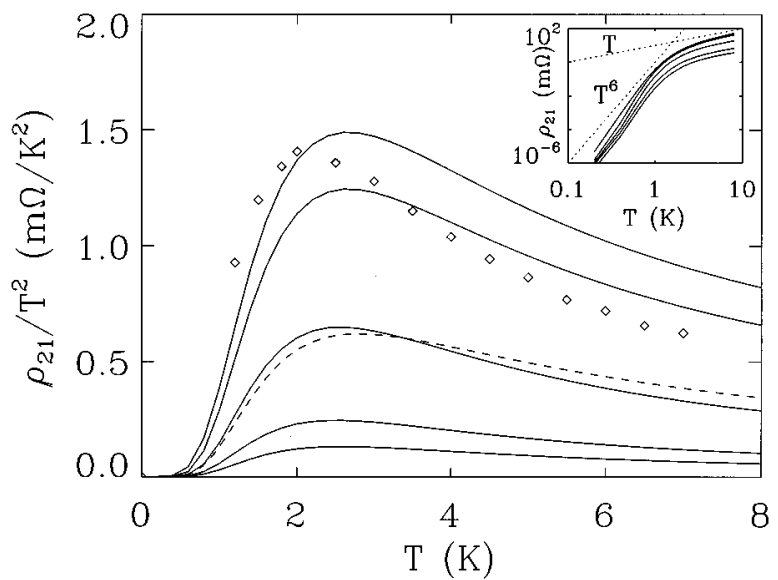

FIG. 3. The scaled transresistivity $\rho_{12} / T^{2}$ as a function of temperature for (in increasing order) $\ell_{\mathrm{ph}}=10^{4}, 10^{5}, 10^{6}, 10^{7}$, and $10^{8} \mathrm{~nm}$. The parameters used are $n=1.5 \times 10^{11} \mathrm{~cm}^{-2}, L=200 \AA$, and $d=500 \AA$ in GaAs. The dots are data points obtained from Ref. 4 , and the dotted line is given by Eq. (43). Inset: Log-log plots for the theoretical curves $\ell_{\mathrm{ph}}=10^{4}$ to $10^{8}$, showing the crossover from $T^{6}$ to $T$ behavior. The dotted lines are for reference.

obtain transparent analytic results, we do not expect them to be exact. In particular, corrections to the random-phase approximation for screening must have some quantitative importance, and are difficult to estimate reliably.

Except where noted, the layer density was chosen to have the typical value $n_{1}=1.5 \times 10^{11} \mathrm{~cm}^{-2}$ which yields a Fermi temperature of $T_{F}=\varepsilon_{F} / k_{B} \simeq 60 \mathrm{~K}$. The GaAs material parameters are taken from the literature, and were given in Sec. III B. ${ }^{33}$

\section{A. Temperature dependence}

As mentioned in Sec. I the transresistivity increases roughly as $T^{2}$ at low temperatures if the effective interaction between the layers is frequency independent. Deviations from this behavior are indicative of retarded effective interactions. In particular, the phonon-mediated contribution to $\rho_{21}$ grows approximately as $T^{10}$ for the deformation potential, and as $T^{6}$ for the piezoelectric contribution. Both cross over to a linear dependence on $T$ at approximately their respective Bloch-Grüneisen temperatures. In Fig. 3, we plot $\rho_{21} / T^{2}$ as a function of $T$. The shape of this curve is a result of an effective interaction which, for each $q$, is sharply peaked as a function of frequency around $\omega=c_{l} q$. This peak in the frequency integral, which produces the dominant contribution to the phonon-mediated drag, is cut off exponentially by the thermal phase-space factor when $\hbar c_{l} q>2 k_{B} T$. This implies that only phonons with energies less than the temperature can participate in the drag. The crossover from $T^{5}$ or $T^{6}$ (where the piezoelectric coupling dominates) to a linear $T$ dependence of $\rho_{21}$ (hence the peak in $\rho_{21} / T^{2}$ ) occurs when the $2 k_{F}$ phonons, which are responsible for most of the momentum transfer in the systems, can be excited. The peak is expected to occur near the temperature scale $T_{\mathrm{BG}}$ $=\hbar 2 c_{l} k_{F} / k_{B}$, which is $7.8 \mathrm{~K}$ for the parameters used in Fig. 3. It in fact occurs at around $T_{\text {peak }} \approx 2.5 \mathrm{~K} \approx T_{\mathrm{BG}} / 3$. For $T$ $\gtrsim T_{\mathrm{BG}}$ the contribution of the deformation-potential phonons
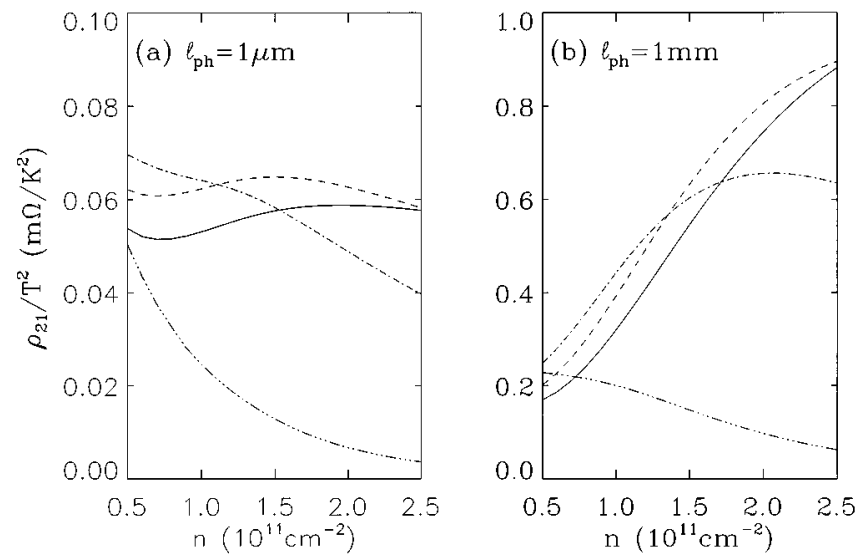

FIG. 4. The transresistivity as a function of matched densities $n$ for (a) $\ell_{\mathrm{ph}}=1 \mu \mathrm{m}$ and (b) $\ell_{\mathrm{ph}}=1 \mathrm{~mm}$. The temperatures are $T$ $=1,2,3$, and $4 \mathrm{~K}$ for dash-triple-dotted, dash-dotted, dashed, and solid lines, respectively. Other parameters as in Fig. 3. The ratio $\ell_{\mathrm{ph}} / \ell_{\mathrm{ph}, \text { crit }}$ varies in (b) from 0.55 for $n=0.5 \times 10^{11} \mathrm{~cm}^{-2}$ to 14.6 for $n=2.5 \times 10^{11} \mathrm{~cm}^{-2}$.

is approximately $50-100$ times greater than that of the piezoelectric phonons for the parameters used in the figure.

We also plot the results of Eq. (43) and the experimental results from Ref. 4. One can see that for the long mean free path approximation, and small layer separation, Eq. (43) underestimates the contribution of the mode. An investigation of the integrand indicates that this is due to a rather long non-Lorenzian tail in the $\omega<c_{l} q$ regime, which can be seen in the solid curve of Fig. 8 discussed below.

Comparison of the magnitude of the experimental points and the theoretical curves seems to indicate that effects of the collective mode have been observed. However, as we caution in Sec. VI D, this should not be construed as definitive proof of the existence of the mode.

\section{B. Density dependence}

From Eqs. (43) and (62) the transresistivity at matched densities is proportional to $1 / n^{2}$ times an integral whose value primarily reflects the allowed phase-space volume for an exchange of phonons with large planar and small perpendicular momenta. The maximum planar momentum transfer $q=2 k_{F}$ is proportional to the square root of the density. At temperatures larger than $T_{\text {peak }}$, the phase-space integral should therefore increase with density, reflecting the larger possible momentum transfers. At temperatures well below $T_{\text {peak }}$, on the other hand, phonons at $q=2 k_{F}$ cannot be exchanged, and a higher-density cannot be exploited. Consequently, in the case where the density is changed at a fixed temperature, the following behavior should be observed. For low densities, the increase in density $n$ permits larger momentum transfers, thus increasing the transresistivity. At some $n$, a further increase does no good as the $2 k_{F}$ wave vectors cannot be excited, and this leads to an overall decrease in $\rho_{21}$. The resulting curve for $\rho_{21}$ has a bump or maximum as a function of the density. The position of the bump or maximum increases with increasing temperature, for the reasons stated above. Figure 4 shows the density dependence of the transresistivity for four different tempera- 

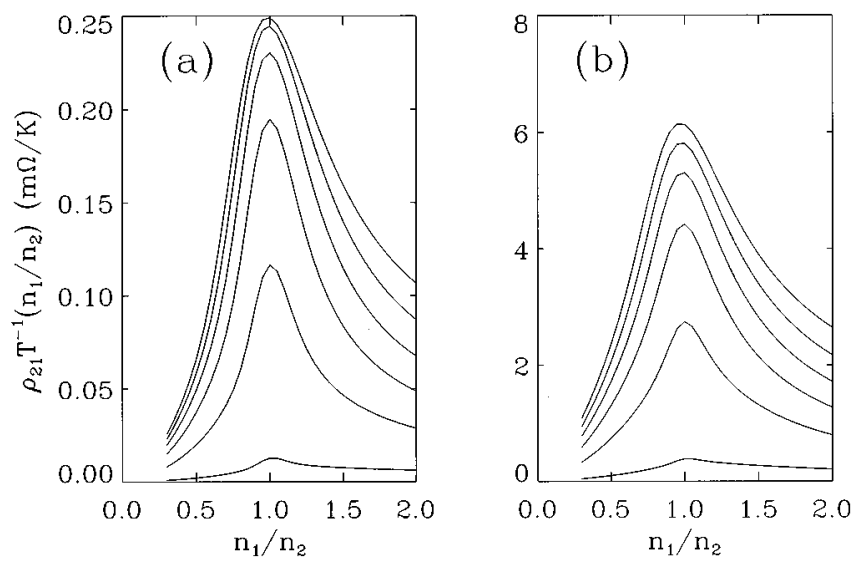

FIG. 5. The scaled transresistivity $\rho_{21} T^{-1} n_{1} / n_{2}$ as a function of the density ratio $n_{1} / n_{2}$ for (in increasing order) $T=1,2,3,4,5$, and $6 \mathrm{~K}, n_{2}=1.5 \times 10^{11} \mathrm{~cm}^{-2}, d=50 \mathrm{~nm}$, (a) $\ell_{\mathrm{ph}}=1 \mu \mathrm{m}$, and (b) $\ell_{\mathrm{ph}}$ $=10 \mathrm{~cm}$. Other parameters are as in Fig. 4 .

tures, $T=1,2,3$, and $4 \mathrm{~K}$ and $\ell_{\mathrm{ph}}=1 \mu \mathrm{m}$ (short mean free path regime) and $1 \mathrm{~mm}$ (coupled-mode-dominated regime).

While the general features discussed above are seen in both regimes, Fig. 4 still shows a qualitative difference between the two. The reason is twofold. A comparison of Eqs. (43) and (62) reveals that there is an extra factor of $\operatorname{Im}[\chi]$ in the integrand of Eq. (62). Since $\operatorname{Im}[\chi]$ decreases with increasing $k_{F}, \rho_{21}$ falls faster with increasing density in the short mean free path regime than in the coupled-mode regime. More importantly, $\ell_{\text {ph,crit }}$ is strongly dependent on density: $\ell_{\text {ph,crit }} \propto\left(2 k_{F}+q_{\mathrm{TF}}\right)^{2} / k_{F}^{5}$. Hence increasing the density can make the coupled mode more and more dominant and lead to an increase of $\rho_{21}$. As seen in Fig. 4, the difference in density dependence could provide a clue regarding which of the two regimes prevails in a particular sample.

\section{Density-ratio dependence}

The transresistivity is strongly dependent on the electrondensity ratio $n_{1} / n_{2}$. For temperatures well below the Fermi temperature, the maximum planar momentum transfer is $q$ $=2 k_{F, \text { min }}$, where $k_{F, \text { min }}$ is the Fermi wave vector of the electron gas with the lowest density. Decreasing one density will lead to a decrease in $\rho_{21}$; a decrease also occurs if one density is increased because of the general $1 / n_{1} n_{2}$ dependence. The resulting peak at equal densities, illustrated in Fig. 5, is independent of temperature and does not occur for the Coulombic drag mechanism (in the absence of any plasmon effects ${ }^{14,16}$ ). This difference was used ${ }^{4}$ to separate Coulomb and phonon drag mechanisms experimentally when the layer separation is sufficiently small that the Coulomb mechanism is of comparable importance.

The calculations were done for both short and long mean free path regimes. However, the shape of the curves are so much alike that it is unlikely that a density-ratio measurement can be used to differentiate these two regimes in an experiment.

\section{Mean free path dependence}

In Sec. VI C we explained that there is a critical $\ell_{\text {ph }}$ beyond which a collective mode appears which enhances the

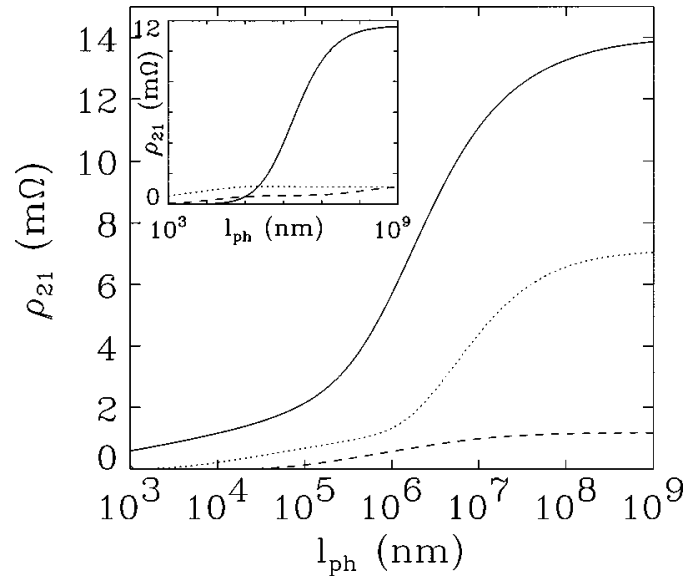

FIG. 6. The transresistivity as a function of the mean free path, for $d=50,3000$, and $5 \times 10^{4} \mathrm{~nm}$ (solid, dotted, and dashed lines, respectively). The density is $1.5 \times 10^{11} \mathrm{~cm}^{-2}$, and $T=3 \mathrm{~K}$. Inset: Real-phonon, virtual-phonon, and coupled-mode contributions (dotted, dashed, and solid lines, respectively) for transresistivity as a function of mean free path, for $d=50 \mathrm{~nm}$ and $T=3 \mathrm{~K}$.

overall transresistivity. In Fig. 6, we plot numerical results for the transresistivity as a function of the mean free path for $T=3 \mathrm{~K}$ and $d=50 \mathrm{~nm}$. For $\ell_{\mathrm{ph}}<\ell_{\mathrm{ph}, \text { crit }}$, we are in the regime where the electron-phonon interaction is separately screened in the two layers, and $\rho_{21}$ increases logarithmically with $\ell_{\mathrm{ph}}$. We see in Fig. 6, when the mean free path exceeds $\ell_{\text {ph,crit }}$ and the collective mode starts to emerge, that the transresistivity increases more rapidly before saturating at a value given roughly by the estimate given in Eq. (43). By splitting the contributions into real $\left(\omega>c_{l} q\right)$, virtual $\left(c_{l} q\right.$ $\left.-\omega \gg \omega_{0}\right)$, and coupled modes $\left(\omega \approx \omega_{0}\right)$, we show in the inset of Fig. 6 that the increase in $\rho_{21}$ which occurs when $\ell_{\text {ph }}$ exceeds $\ell_{\text {ph,crit }}$ comes mainly from the coupled-mode contribution.

In the low-temperature regime where the drag experiments were performed, $\ell_{\mathrm{ph}}$ is certainly sample dependent, but should be temperature independent for a given sample. Extrapolating the logarithmic $\ell_{\mathrm{ph}}$ dependence (i.e., which neglects effects of collective screening) in Fig. 6 for the $d$ $=50 \mathrm{~nm}$ case, we find that the value of $\ell_{\mathrm{ph}}$ required to fit measured values ${ }^{4}$ of $\rho_{21}(\approx 12 \mathrm{~m} \Omega)$ are (literally) astronomical in magnitude. This could be taken as evidence of the importance of cooperative screening and collective modes in present experimental systems. On the other hand, our numerical calculations are dependent on random-phaseapproximation estimates of $\operatorname{Im} \chi$, which are likely to require substantial numerical revision ${ }^{34}$ especially in the important region near $q=2 k_{F}$. These corrections will increase $\operatorname{Im} \chi$, and could possibly boost the theoretically calculated $\rho_{21}$ to experimental values without invoking the coupled electronphonon mode. The random-phase approximation for the screening function can also lead to a quantitative discrepancy. Hence we do not claim that there is incontrovertible experimental evidence for the existence of the coupled electron-phonon mode.

\section{E. Layer separation dependence}

It is possible to prepare a series of double-quantum-well systems which are substantially identical apart from the 


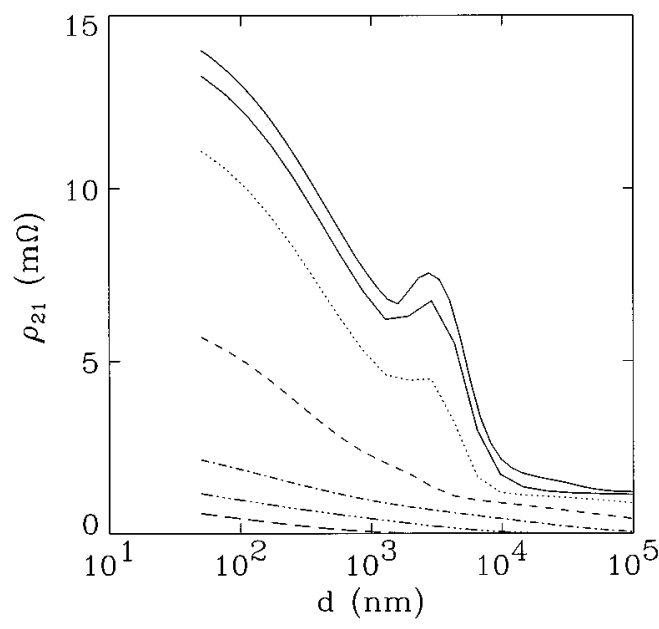

FIG. 7. The transresistivity as a function of the well separation for (in increasing order) $\ell_{\mathrm{ph}}=10^{3}, 10^{4}, 10^{5}, 10^{6}, 10^{7}$, and $10^{8} \mathrm{~nm}$, at $T=3 \mathrm{~K}, n=1.5 \times 10^{11} \mathrm{~cm}^{-2}$, and $L=200 \AA$.

separation between the 2D electron layers. ${ }^{4,35}$ Experiments on such a series of samples will give a more certain indication of the operative phonon exchange regime than experiments on a single sample.

In Fig. 7 we plot the transresistivity as a function of the separation between the wells, for various phonon mean free paths. For $\ell_{\mathrm{ph}}$ small enough that the collective mode has not developed, $\rho_{21}$ exhibits a purely logarithmic dependence on $d$, until $d$ exceeds $\ell_{\mathrm{ph}} /\left(2 k_{F} L\right)$, when it begins to fall exponentially. For $\ell_{\mathrm{ph}} \gtrsim \ell_{\mathrm{ph} \text {,crit }}$, the behavior is more complex. The collective mode develops, and $\rho_{21}$ is considerably enhanced. For small $d$, our numerical results suggest that $\rho_{21}$ also decreases logarithmically with $d$, in contradiction with the $d$ independence implied in Eq. (43). Figure 8, which shows the integrand of the transresistivity expression as a

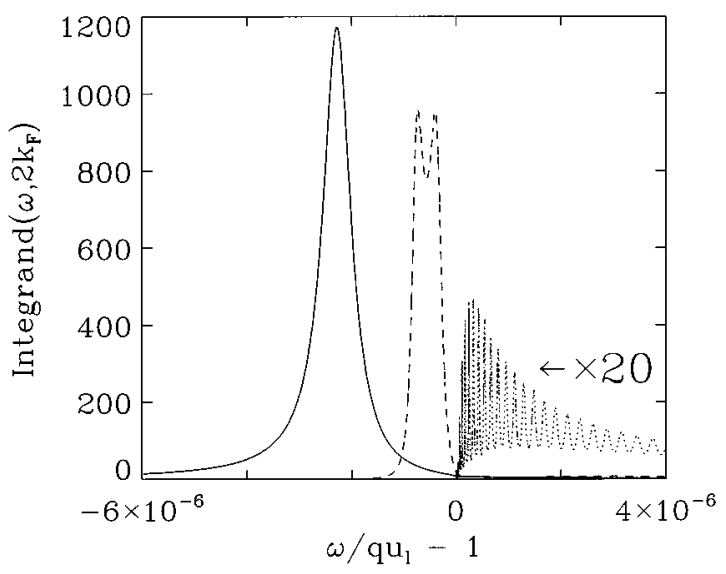

FIG. 8. The integrand as a function of $\omega$ for $q=2 k_{F}$ and $\ell_{\mathrm{ph}}$ $=\infty$, for $d=50 \mathrm{~nm}$ (solid), $d=3 \times 10^{3} \mathrm{~nm}$ (dashed) and $d$ $=5 \times 10^{4} \mathrm{~nm}$ (dotted). Other parameters are as in Fig. 7. As the well separation increases, and the electron-phonon mode decouples into two weakly coupled individual modes, leading to the twinpeaked structure at $d=3 \times 10^{3} \mathrm{~nm}$. At large well separations, the collective modes do not contribute to the transresistivity, and the entire momentum transfer is due to the real phonons. function of frequency at $q=2 k_{F}$, indicates that there is a substantial non-Lorenzian tail for $\omega<c_{l} q-\omega_{0}$, which is not taken into account in the analysis leading to Eq. (43). This tail contributes to the nearly logarithmic $d$ dependence in the regime where the collective mode contributes significantly.

Another surprising result is that, in this regime, $\rho_{21}$ does not decrease monotonically as $d$ is increased. Instead there is

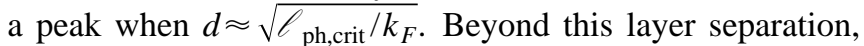
the electron-phonon collective mode of the double quantum well begins to decouple into two weakly coupled modes centered around each well. This is illustrated in Fig. 8, where we compare the frequency integrands for $d<\sqrt{\ell_{\mathrm{ph}, \mathrm{crit}} / k_{F}}$ and $d$ $>\sqrt{\ell_{\mathrm{ph}, \text { crit }} / k_{F}}$. As $d$ is increased further beyond $\sqrt{\ell_{\mathrm{ph}, \text { crit }} / k_{F}}$, the transresistivity eventually resumes its decline.

\section{SUMMARY AND CONCLUSIONS}

Phonon exchange contributes importantly to the frictional drag resistance between nearby electron layers, and is the dominant drag mechanism at large two-dimensional layer separations. In this paper we reported on a thorough theoretical analysis of this drag mechanism. We find that drag includes contributions due to exchange of both virtual and real phonons, and that coupled collective modes of the 2D electron and 3D phonon systems can play a role depending on sample geometry and material parameters. We distinguish two regimes based on the relationship between the phonon mean free path and a crossover length scale $\ell_{\text {ph,crit }}$ which is typically of the order of $0.2 \mathrm{~mm}$. It is possible that the mean free path for high quality molecular-beam epitaxy grown heterostructures can exceed $\ell_{\text {ph,crit }}$ at low temperatures.

In the short mean free path regime the dominant drag processes at momentum transfer $q$ have an energy transfer just below $c_{l} q$ for virtual phonons, and just above $c_{l} q$ for real phonons. The drag rate in this case decreases logarithmically with layer separation $d$, until $d$ reaches $d_{a}$ $=\ell_{\mathrm{ph}} / 2 k_{F} L \sim \ell_{\mathrm{ph}}$. The weak layer separation dependence comes nearly entirely from the virtual-phonon exchange contribution. For $d>d_{a}$, the virtual-phonon exchange contribution is small and the real-phonon exchange contribution decreases exponentially with layer separation. The real-phonon exchange contribution to the drag is consistent with expectations based on the coupled Boltzmann equations for the electron and phonon systems, and the exponential falloff for $d$ $>d_{a}$ can be understood in terms of the decay length for the disturbance of the steady-state phonon system as the result of current flowing in one of the electron layers.

For samples with phonon mean free paths larger than $\ell_{\text {ph,crit }}$, a collective mode involving both electronic and lattice degrees of freedom emerges below the continuum of 3D phonon energies with 2D wave vectors $q$ near $2 k_{F}$. The existence of this mode enhances the drag. In this regime the drag also has a roughly logarithmic layer separation, until $d$ reaches another crossover length $d_{B}=\left(1+q_{\mathrm{TF}} / 2 k_{F}\right) /$ $16 k_{F} C_{\mathrm{DP}}$. For typical samples, $d_{B} \sim 0.5 \mu \mathrm{m}$. At this layer separation the collective mode separates into weakly coupled modes associated with the individual 2D layers. Although the drag has a complicated and nonmonotonic layer separation in this regime, it does ultimately decline with increasing $d$. These findings are summarized in Table I.

There are, unfortunately, few existing experiments on the 
TABLE I. Table summarizing the distance behavior for the collective-mode regime and for the damped-phonon cases.

\begin{tabular}{l|c|c|c|c}
\hline \hline \multicolumn{1}{c|}{$\ell_{\mathrm{ph}}$} & \multicolumn{2}{|c|}{$\ell_{\mathrm{ph}} \ll \ell_{\mathrm{ph}, \mathrm{crit}}$} & \multicolumn{2}{|c}{$\ell_{\mathrm{ph}} \gg \ell_{\mathrm{ph}, \mathrm{crit}}$} \\
\hline \multicolumn{1}{c|}{ Physics } & \multicolumn{2}{|c|}{ Damped phonons } & \multicolumn{2}{|c}{ Coupled $e$-ph mode } \\
\hline$d$ & $d \ll d_{a}$ & $d \gg d_{a}$ & $d \ll d_{B}$ & $d \gg d_{B}$ \\
\cline { 2 - 5 } $\begin{array}{l}\text { Distance } \\
\text { dependence }\end{array}$ & $\ln \left(d_{a} / d\right)$ & $\frac{d_{a}}{d} \exp \left(-d / d_{a}\right)$ & $\begin{array}{c}\text { Weak } \\
(\text { logarithmic })\end{array}$ & Complicated \\
\hline \hline
\end{tabular}

long distance dependence of the phonon-mediated drag resistivity. More experimental work will be needed to understand the range of possible behaviors and their dependence on system parameters. We note, however, that the preliminary experimental results by Gramila et $a .^{35}$ are consistent with a logarithmic $d$ dependence.

\section{ACKNOWLEDGMENTS}

This work was supported in part by the National Science Foundation under Grant No. DMR-9416906. M. C. B. was supported by the Danish Research Academy. The authors acknowledge helpful conversations with Werner Dietche, Jim Eisenstein, Tom Gramila, Antti-Pekka Jauho, M. Reizer, Holger Rubel, and Ned Wingreen.

\section{APPENDIX: BOLTZMANN EQUATION DERIVATION OF THE PHONON DRAG}

A sketch of the derivation of the contribution due to real emission of phonons is given here. This Boltzmann equation approach has been used previously to estimate the real phonon contribution to the drag. 4,36

Assume that the distribution function of the driving layer is a drifted Fermi Dirac. Then the deviation function $\psi_{i}(\mathbf{k})$ $\equiv \delta f_{0, i}(\mathbf{k}) /\left[\left(1-f_{0, i}(\mathbf{k})\right) f_{0, i}(\mathbf{k})\right]$ (where $f_{0, i}$ is the equilibrium distribution function in layer $i$ ) for a driving field $e \mathbf{E}_{1}$ in the $x$ direction is

$$
\psi_{1}(\mathbf{k})=\frac{\tau e E_{1}}{k_{B} T} v_{x}(\mathbf{k})
$$

The generation of nonequilibrium phonons is given by the electron-phonon coupling, and is

$$
\begin{aligned}
&\left(\frac{\partial N(\mathbf{Q})}{\partial t}\right)_{\text {gen }}=-\frac{2 \pi}{\hbar} \frac{2}{V} \sum_{k}\left|g_{1}(Q)\right|^{2} \delta\left(\varepsilon_{\mathbf{k}+\mathbf{q}}-\left[\varepsilon_{\mathbf{k}}+\hbar \omega_{\mathbf{Q}}\right]\right) \\
& \times f_{0,1}(\mathbf{k})\left[1-f_{0,1}(\mathbf{k}+\mathbf{q})\right] N_{0}(\mathbf{Q}) \\
& \times\left[\psi_{1}(\mathbf{k})-\psi_{1}(\mathbf{k}+\mathbf{q})+\Phi(\mathbf{Q})\right], \\
& \Phi(\mathbf{Q})=\delta N(\mathbf{Q}) /\left\{N_{0}(Q)\left[1+N_{0}(Q)\right]\right\},
\end{aligned}
$$

where $\delta N$ is the nonequilibrium distribution of phonons, and $N_{0}(Q)=\left[\exp \left(\hbar \omega_{Q} / k_{B} T\right)-1\right]^{-1}$. The coupling constant $g_{1}(\mathbf{Q})$ is given by

$$
g_{1}(\mathbf{Q})=|M(\mathbf{Q})|^{2}\left|F_{1}\left(Q_{z}\right)\right|^{2}
$$

Since the phonon coupling constant is small, we ignore the $\Phi(\mathbf{Q})$ term in Eq. (A2) because it is higher order in $g$.

Then, one can write the phonon generation rate (using the identity $f_{0}(\epsilon)\left[1-f_{0}(\epsilon+\omega)\right]=\left[f_{0}(\epsilon)-f_{0}(\epsilon+\omega)\right]\left[N_{0}(\omega)\right.$ $+1]$ )

$$
\begin{aligned}
\left(\frac{\partial N(\mathbf{Q})}{\partial t}\right)_{\text {gen }}= & -\frac{2}{L_{z}}|g(\mathbf{Q})|^{2} N_{0}(Q)\left[1+N_{0}(Q)\right] \\
& \times \frac{q \tau e E_{1}}{m^{*} k_{B} T} \operatorname{Im} \chi\left(q, \omega_{\mathbf{Q}}\right) .
\end{aligned}
$$

The phonon Boltzmann equation is

$$
\begin{aligned}
v_{z} \frac{\partial \Phi(\mathbf{Q}, z)}{\partial z}= & \delta(z) 2|g(\mathbf{Q})|^{2} \frac{q_{x} \tau e E_{1}}{m^{*} k_{B} T}\left[-\operatorname{Im} \chi\left(q, \omega_{\mathbf{Q}}\right)\right] \\
& -\frac{\Phi(\mathbf{Q}, z)}{\tau_{\mathrm{ph}}}
\end{aligned}
$$

whose solution is

$$
\begin{aligned}
\Phi(\mathbf{Q}, z)= & -\frac{2\left|g_{1}(\mathbf{Q})\right|^{2} q_{x} \tau e E_{1}}{m^{*} k_{B} T\left|v_{z}(\mathbf{Q})\right|} \operatorname{Im} \chi_{1}\left(q, \omega_{Q}\right) \\
& \times \exp \left(-\left|\frac{z}{v_{z}(\mathbf{Q}) \tau_{\mathrm{ph}}}\right|\right) \theta\left(z Q_{z}\right) .
\end{aligned}
$$

The electron-phonon collision term in layer 2 is

$$
\begin{aligned}
\left(\frac{\partial f}{\partial t}\right)_{2}= & -\frac{2 \pi}{\hbar} \frac{2}{V} \sum_{\mathbf{Q}}\left|g_{2}(\mathbf{Q})\right|^{2}\left\{-\delta\left(\varepsilon_{\mathbf{k}+\mathbf{q}}-\varepsilon_{\mathbf{k}}+\hbar \omega_{\mathbf{Q}}\right)\right. \\
& \times\left[1+N_{0}(\mathbf{Q})\right] \Phi(-\mathbf{Q})+\delta\left(\varepsilon_{\mathbf{k}+\mathbf{q}}-\varepsilon_{\mathbf{k}}-\hbar \omega_{\mathbf{Q}}\right) \\
& \left.\times N_{0}(\mathbf{Q}) \Phi(\mathbf{Q})\right\}
\end{aligned}
$$

Using $\Phi(-\mathbf{Q})=-\Phi(\mathbf{Q})$, the total momentum transfer to the second layer is 


$$
\left(\frac{\partial \mathbf{p}}{\partial t}\right)_{2}=2 \pi \int \frac{d \mathbf{q}}{(2 \pi)^{2}} \frac{\mathbf{q} q_{x} \tau e E_{1}}{m^{*} k_{B} T} \int_{0}^{\infty} \frac{d Q_{z}}{2 \pi} \frac{2\left|g_{1}(\mathbf{Q})\right|^{2}\left|g_{2}(\mathbf{Q})\right|^{2}}{v_{z}(\mathbf{Q})} \frac{\exp \left[-d / v_{z}(\mathbf{Q}) \tau_{\mathrm{ph}}\right]}{4 \sinh ^{2}\left(\hbar \omega_{Q} / 2 k_{B} T\right)} \operatorname{Im} \chi_{1}\left(q, \omega_{Q}\right) \operatorname{Im} \chi_{2}\left(q, \omega_{Q}\right)
$$

Given that $\omega_{Q}=c Q$, the transresistivity can be expressed in the following form:

$$
\begin{aligned}
\rho_{21}= & \frac{E_{2}}{J_{1}}=\frac{-\left(d p_{2, x} / d t\right) m^{*}}{e^{2} n_{1} n_{2} E_{1} \tau}=\frac{-1}{8 \pi^{2} e^{2} n_{1} n_{2} k_{B} T} \int_{0}^{\infty} d q q^{3} \\
& \times \int_{0}^{\infty} d \omega \frac{\operatorname{Im} \chi_{1}(q, \omega) \operatorname{Im} \chi_{2}(q, \omega)}{\sinh ^{2}\left(\hbar \omega / 2 k_{B} T\right)} \frac{\omega^{2}\left|F_{1}\left(\sqrt{\left(\omega / c_{l}\right)^{2}-q^{2}}\right)\right|^{2}\left|F_{2}\left(\sqrt{\left(\omega / c_{l}\right)^{2}-q^{2}}\right)\right|^{2}\left|M\left(q, \sqrt{(\omega / c)^{2}-q^{2}}\right)\right|^{4}}{c_{l}^{2}\left[\omega^{2}-\left(q c_{l}\right)^{2}\right]} \\
& \times \exp \left(-\frac{d}{c_{l} \tau_{\mathrm{ph}} \sqrt{1-c_{l}^{2} q^{2} / \omega^{2}}}\right) .
\end{aligned}
$$

In comparison, the expression for the "real" contribution to the transresistivity is

$$
\begin{aligned}
\rho_{21}= & \frac{-\hbar^{2}}{8 \pi^{2} e^{2} n_{1} n_{2} k_{B} T} \int_{0}^{\infty} d q q^{3} \\
& \times \int_{0}^{\infty} d \omega\left[\mathcal{D}_{21, \text { real }}\right]^{2} \frac{\operatorname{Im} \chi_{1}(q, \omega) \operatorname{Im} \chi_{2}(q, \omega)}{\sinh ^{2}\left(\hbar \omega / 2 k_{B} T\right)},
\end{aligned}
$$

where

$$
\begin{aligned}
\mathcal{D}_{21, \text { real }}(\mathbf{q}, \omega)= & \int_{-\infty}^{\infty} \frac{d q_{z}}{2 \pi \hbar}\left|M\left(q, q_{z}\right)\right|^{2} F_{1}\left(Q_{z}\right) F_{2}\left(-Q_{z}\right) \\
& \times \operatorname{Im} D(\mathbf{Q}, \omega), \\
\operatorname{Im} D(\mathbf{Q}, \omega)= & {\left[\frac{1 / 2 \tau_{\mathrm{ph}}}{\left(\omega+c_{l} \sqrt{q^{2}+q_{z}^{2}}\right)^{2}+\left(1 / 2 \tau_{\mathrm{ph}}\right)^{2}}\right] } \\
& -\left[\frac{1 / 2 \tau_{\mathrm{ph}}}{\left(\omega-c_{l} \sqrt{q^{2}+q_{z}^{2}}\right)^{2}+\left(1 / 2 \tau_{\mathrm{ph}}\right)^{2}}\right] .
\end{aligned}
$$

For small $1 / \tau_{\mathrm{ph}}$, the poles of the Lorenzian are

$$
\begin{aligned}
q_{z} & = \pm \sqrt{\left[\omega+i /\left(2 c_{l} \tau\right)\right]^{2}-q^{2}}, \\
& \approx \pm \sqrt{\omega^{2} / c_{l}^{2}-q^{2}} \pm i \frac{\omega}{2 \tau c_{l}^{2} \sqrt{\omega^{2} / c_{l}^{2}-q^{2}}} .
\end{aligned}
$$

As they lie close to the real axis, the Lorenzians can in general be approximated by $\delta\left(\omega \pm c_{l} \sqrt{q^{2}+q_{z}^{2}}\right)$. The imaginary part of the pole, however, does affect the $\exp \left(-i Q_{z} d\right)$ term which comes from the difference in the phases of the form factors $F_{1}\left(Q_{z}\right)$ and $F_{2}\left(-Q_{z}\right)$. The $Q_{z}$ integration results in the insertion of the imaginary part of the pole into the exponent, yielding

$$
\begin{aligned}
\mathcal{D}_{21, \text { real }}(\mathbf{q}, \omega) \approx & \frac{-1}{\hbar}\left|M\left(q, \sqrt{\omega^{2} / c_{l}^{2}-q^{2}}\right)\right|^{2} F_{1}\left(\sqrt{\omega^{2} / c_{l}^{2}-q^{2}}\right) \\
& \times F_{2}\left(\sqrt{\omega^{2} / c_{l}^{2}-q^{2}}\right) \frac{\omega}{c_{l}^{2} \sqrt{\omega^{2} / c_{l}^{2}-q^{2}}} \\
& \times \exp \left(-\frac{d}{2 c_{l} \tau_{\mathrm{ph}} \sqrt{1-c_{l}^{2} q^{2} / \omega^{2}}}\right) \cdot
\end{aligned}
$$

Substituting Eq. (A14) into Eq. (A11) gives Eq. (A10).
${ }^{1}$ M. B. Pogrebinskii, Fiz. Tekh. Poluprovodn. 11, 637 (1977) [Sov. Phys. Semicond. 11, 372 (1977)]; P. J. Price, Physica B\&C 117, 750 (1983).

${ }^{2}$ P. M. Solomon, P. J. Price, D. J. Frank, and D. C. La Tulipe, Phys. Rev. Lett. 63, 2508 (1989).

${ }^{3}$ T. J. Gramila, J. P. Eisenstein, A. H. MacDonald, L. N. Pfeiffer, and K. W. West, Phys. Rev. Lett. 66, 1216 (1991); Surf. Sci. 263, 446 (1992).

${ }^{4}$ T. J. Gramila, J. P. Eisenstein, A. H. MacDonald, L. N. Pfeiffer, and K. W. West, Phys. Rev. B 47, 12957 (1993); Physica B 197, 442 (1994).

${ }^{5}$ U. Sivan, P. M. Solomon, and H. Shtrikman, Phys. Rev. Lett. 68, 1196 (1992).

${ }^{6}$ H. C. Tso, P. Vasilopoulos, and F. M. Peeters, Phys. Rev. Lett.
70, 2146 (1993); L. Swierkowski, J. Szymanski, and Z. W. Gortel, ibid. 74, 3245 (1995); G. Vignale, and A. H. MacDonald, ibid. 76, 2786 (1996).

${ }^{7}$ B. Laikhtman and P. M. Solomon, Phys. Rev. B 41, 9921 (1990); I. I. Boiko and Yu. M. Sirenko, Phys. Status Solidi B 159, 805 (1990); P. M. Solomon and B. Laikhtman, Superlattices Microstruct. 10, 89 (1991); A. G. Rojo and G. D. Mahan, Phys. Rev. Lett. 68, 2074 (1992); D. I. Maslov, Phys. Rev. B 45, 1911 (1992); H. L. Cui, X. L. Lei, and N. J. M. Horing, Superlattices Microstruct. 13, 221 (1993); L. Świerkowski, J. Szymański, and Z. W. Gortel, Phys. Rev. Lett. 74, 3245 (1995).

${ }^{8}$ Martin Mosko, Vladimir Cambel, and Antonia Moskova, Phys. Rev. B 46, 5012 (1992); Vladimir Cambel and Martin Mosko, Semicond. Sci. Technol. 8, 364 (1993). 
${ }^{9}$ H. C. Tso, P. Vasilopoulos, and F. M. Peeters, Phys. Rev. Lett. 68, 2516 (1992).

${ }^{10}$ C. Zhang and Y. Takahashi, J. Phys.: Condens. Matter 5, 5009 (1993).

${ }^{11}$ A.-P. Jauho and H. Smith, Phys. Rev. B 47, 4420 (1993).

${ }^{12}$ Lian Zheng and A. H. MacDonald, Phys. Rev. B 48, 8203 (1993).

${ }^{13}$ A. Kamenev and Y. Oreg, Phys. Rev. B 52, 7516 (1995).

${ }^{14}$ K. Flensberg and B. Y.-K. Hu, Phys. Rev. Lett. 73, 3572 (1994).

${ }^{15}$ K. Flensberg, B. Y.-K. Hu, A.-P. Jauho, and J. Kinaret, Phys. Rev. B 52, 14761 (1995).

${ }^{16}$ K. Flensberg and B. Y.-K. Hu, Phys. Rev. B 52, 14796 (1995).

${ }^{17}$ L. Świerkowski, J. Szymański, and Z. W. Gortel, Phys. Rev. B 55, 2280 (1997).

${ }^{18}$ H. Rubel, E. H. Linfield, D. A. Ritchie, K. M. Brown, M. Pepper, and G. A. C. Jones, Semicond. Sci. Technol. 10, 1229 (1995).

${ }^{19}$ A. H. MacDonald, Phys. Rev. Lett. 44, 489 (1980); M. Kaveh and N. Wiser, Adv. Phys. 33, 257 (1984); K. Schwartzman and W. E. Lawrence, Phys. Rev. B 48, 14089 (1993).

${ }^{20}$ K. Hubner and W. Shockley, Phys. Rev. Lett. 4, 504 (1960).

${ }^{21}$ P. J. Price, Physica B \& C 134, 164 (1985); M. Mosko, J.-L. Pelouard, and F. Pardo, Phys. Rev. B 52, 5830 (1995).

${ }^{22}$ N. P. R. Hill et al., Phys. Rev. Lett. 78, 2204 (1997).

${ }^{23}$ T. J. Gramila, Bull. Am. Phys. Soc. 42, 54 (1997).

${ }^{24}$ In our calculation we follow the common practice of treating the phonon system as uniform. There is actually a difference of $\sim 5 \%$ between sound velocities of GaAs and $\mathrm{Al}_{x} \mathrm{Ga}_{1-x}$ As for typical values of $x$. Although we have not performed detailed calculations which respect this mismatch, we do not believe that doing so would materially alter the conclusions reached in this paper.

${ }^{25}$ G. D. Mahan, Many-Particle Physics, 2nd ed. (Plenum, New York, 1990).

${ }^{26}$ The interaction Hamiltonian entering Eq. (8b) should be the sum of $\hat{H}_{e \text {-ph }}$ [see Eq. (3)] and a Coulomb interaction Hamiltonian. The dominating term in the resulting formula for the transconductivity is of the form of Eq. (16), but with $\mathcal{D}_{21}$ replaced by $\mathcal{D}_{21}+U_{21}$.

${ }^{27}$ P. J. Price, Ann. Phys. (N.Y.) 133, 217 (1981).

${ }^{28}$ S. K. Lyo, Phys. Rev. B 38, 6345 (1988).

${ }^{29}$ See for example, Sec. 43 of L. D. Landau and E. M. Lifshitz, Quantum Mechanics, 3rd ed. (Pergamon, Oxford, 1977).

${ }^{30}$ A. B. Migdal, Zh. Eksp. Teor. Fiz. 34, 1438 (1958) [Sov. Phys. JETP 7, 996 (1958)].

${ }^{31}$ T. Holstein, Ann. Phys. (N.Y.) 29, 410 (1964).

${ }^{32}$ Philip B. Allen and Richard Silberglitt, Phys. Rev. B 9, 4733 (1974).

${ }^{33}$ H. L. Stormer, L. N. Pfeiffer, K. W. Baldwin, and K. W. West, Phys. Rev. B 41, 1278 (1990).

${ }^{34}$ At typical 2D electron densities corrections to the random-phase approximation for the real static density-density response function are quite important at $q \sim 2 k_{F}$. It follows from KronigKramers considerations that the same must be true for the imaginary part of the dynamic response function. See S. Moroni, D. M. Ceperley, and G. Senatore, Phys. Rev. Lett. 75, 689 (1995).

${ }^{35}$ S. Zelakiewicz, H. Noh, T. J. Gramila, L. N. Pfeiffer, and K W. West, Bull. Am. Phys. Soc. 42, 485 (1997).

${ }^{36}$ T. J. Gramila (unpublished). 